Preprint typeset in JHEP style - HYPER VERSION

SISSA $61 / 2010 / \mathrm{EP}$

ULB-TH/10-30

hep-th/1009.4158

\title{
Relevant Deformations in Open String Field Theory: a Simple Solution for Lumps
}

\author{
L.Bonora \\ International School for Advanced Studies (SISSA) \\ Via Bonomea 165, 34136 Trieste, Italy, and INFN, Sezione di Trieste \\ E-mail: bonora@sissa.it,

\section{C.Maccaferri} \\ International Solvay Institutes and Physique Théorique et Mathématique, \\ ULB C.P. 231, Université Libre de Bruxelles, B-1050, Bruxelles, Belgium \\ E-mail: maccafer@gmail.com, \\ D.D.Tolla \\ Department of Physics and University College, Sungkyunkwan University, Suwon \\ 440-746, South Korea \\ E-mail: ddtolla@skku.edu
}

ABStRACT: We propose a remarkably simple solution of cubic open string field theory which describes inhomogeneous tachyon condensation. The solution is in one-to-one correspondence with the IR fixed point of the RG-flow generated in the two-dimensional worldsheet theory by integrating a relevant operator with mild enough OPE on the boundary. It is shown how the closed string overlap correctly captures the shift in the closed string one point function between the UV and the IR limits of the flow. Examples of lumps in non-compact and compact transverse directions are given.

KEYwords: String Field Theory. 


\section{Contents}

1. Introduction and outlook 2

2. Reparametrizations of the Erler-Schnabl Tachyon Vacuum 5

3. The solution: general structure 8

3.1 Adding a relevant field to the $K, B, c$ algebra 8

3.2 Solution from pure gauge ansatz 11

3.3 Is this different from the Tachyon Vacuum? 14

4. A class of nontrivial solutions 14

5. Closed String overlap 16

6. Energy, a general expression for the cubic term 18

7. Examples 20

7.1 An explicit example: lumps in non-compact dimensions 20

7.1.1 Multiple lower dimensional branes? 22

7.2 Other examples: primary seeds 23

7.2.1 Interpolating from infinite to finite volume

8. Conclusion and discussion $\quad 26$

A. Building the solution from the Tachyon Vacuum 29

A.1 Seeding the D25-brane 30

A.2 Seeding a lower dimensional brane 31

B. Explicit real form for the energy 32

G. Cubic Superstring generalization 34

D. Computations for the : $X^{2}:$ deformation 35

D.1 Explicit results for one-point function 37

D.2 Three-point functions: energy 37

E. Ordered exponential insertion 40 


\section{Introduction and outlook}

It is expected that Witten's covariant cubic string field theory, [1], has classical solutions describing Boundary Conformal Field Theories (BCFT) which are different from the one we use to define it. This expectation has been checked by the explicit construction of analytic

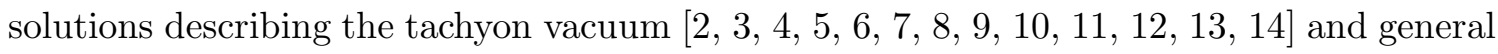
marginal boundary deformations of the initial BCFT [15, 16, 17, 18, 19, 20, 21, 22, 23, 24. The well known worldsheet description of such systems is now analytically understood in the context of cubic OSFT (see [25, 26] for review). This is mainly due to the progress made in identifying very simple (differential) subalgebras of the $*$-product, such as the one of wedge states with insertions [27, 28, 29, 30, 31].

There are other situations which are pretty well understood from a purely worldsheet perspective and we would like to be able to describe within OSFT: relevant boundary deformations of the initial BCFT. While marginal deformations describe the moduli space of the theory around the perturbative vacuum (thus deforming the worldsheet field theory while preserving conformal invariance), relevant deformations are drastically different in that they correspond to unstable directions in the string-field potential which might (or might not) lead to a new vacuum with less space-time energy than the original one. On the worldsheet, they break conformal invariance at the boundary and thus trigger an RG flow to a new BCFT, which has the same bulk content, but different boundary conditions. Moreover, the loss of space-time energy is encoded in the boundary entropy (the log of the disk partition function), which according to the $g$-theorem/conjecture decreases along the RG flow 32]. Actually the tachyon vacuum is the simplest of such RG flows as it corresponds to a trivial BCFT where all the boundary degrees of freedom have been killed. This IR fixed point is achieved by integrating over the worldsheet boundary a constant (positive) tachyon vertex operator. Different, less trivial, IR fixed points can be reached by giving a profile to the tachyon. In this case one expects to get lower dimensional D-branes, placed at the minima of the boundary world-sheet potential (which is just the tachyon profile) [33, 34].

With this picture in mind, we will propose a very simple solution of the OSFT equation of motion which is meant to describe the IR fixed point of a relevant boundary deformation of the initial $B C F T_{0}$, where the tachyon (which is the only matter field which can be relevant) is allowed to have a non trivial space-time dependence. There has been evidence since the seminal work of Moeller, Sen and Zwiebach, [35], where a lower dimensional Dbrane has been seen to emerge as inhomogeneous tachyon condensation in the modified level truncation scheme in Siegel gauge, that these solutions should exist. However an analytic expression for them (in a suitable gauge) has not yet been found.

Here, inspired by the original proposal of Ellwood [39], we give a concrete construction for such a solution. It should be stressed, however, that (differently from the numerical analysis of [35]) we do not provide a way to solve the RG flow using OSFT but, less ambitiously, we explicitly show that whenever the RG flow is known on the worldsheet, a simple solution of OSFT can be written down which represents the IR fixed point of the flow, reproducing the correct coupling with on-shell closed strings. While, at this stage, 
this does not immediately give new tools to explore unknown regions of the open string landscape, we believe it is still important in keeping with the paradigm that OSFT is a consistent and complete space-time theory that can describe such a landscape.

The solution is associated to a matter relevant vertex operator $\phi_{u}(z)$. Here $u$ parametrizes the RG flow obtained by integrating $\phi_{u}$ on the boundary of the unit disk,

$$
S^{(u)}=S_{0}+\int_{\partial D i s k} d \theta \phi_{u}(\theta)
$$

with $S_{0}$ corresponding to the original BCFT and with the conventions that $u=0$ corresponds to the UV (that is $\phi_{u=0}=0$ ) and $u=\infty$ to the IR, which is possible for all the explicit cases we consider.

In order to avoid the subtle issue of renormalization of UV divergences (which occur for general tachyon profiles) we will consider the case in which the relevant field $\phi_{u}$ is subject to the condition

$$
\left(c \phi_{u}\right)^{2}=0
$$

where $c$ is the reparametrization ghost. If $\phi_{u}$ were a marginal field, the above condition would rule out any non trivial OPE of $\phi_{u}$ with itself. In our case, however, the field $\phi$ can have divergent OPE and still satisfy (1.2). This is because if $\phi$ is relevant enough, its contact term divergence can be weaker than the vanishing of the $c c$ collision. In the case where $\phi$ is made of primary operators

$$
\phi=\sum_{i} \chi_{i}^{\left(h_{i}\right)}
$$

this is realized whenever all of them have weights

$$
h_{i}<\frac{1}{2}
$$

see [36] for an explicit example. The region $\frac{1}{2} \leq h<1$ should be explored with a consistent renormalization method as in the case of marginal deformations, [17]. We do not try to attack this problem in the present paper.

The solution can be written within a minimal extension of the universal $K, B, c$ algebra, [3, 8, 9] where we also add the identity-based insertion (defined in the $\frac{2}{\pi}$ arctan-sliver frame)

$$
\phi_{u}=\phi_{u}\left(\frac{1}{2}\right) I
$$

where $I$ is the identity string field. It takes the form

$$
\psi_{u}=c \phi_{u}-\frac{B}{K+\phi_{u}} u \partial_{u} \phi_{u} c \partial c
$$

The $u$ dependence turns out to be a gauge artifact as $u$ can be changed by a simple scale transformation in the sliver frame (which is a midpoint preserving reparametrization). The structure is similar to a reparametrization of the Erler-Schnabl Tachyon Vacuum (TV), [4]

$$
\psi_{T V}^{u}=\frac{u}{u+K} c(u+K) B c=u c-\frac{u}{u+K} B c \partial c, \quad u>0,
$$


which, for reparametrization parameter $u=1$, reduces to (the 'left' version of) [4]. In particular the tachyon vacuum corresponds to the simplest choice of $\phi_{u}$

$$
\phi_{u}^{(T V)}=u .
$$

We show that if the string field $\frac{1}{K+\phi_{u}}$ is regular, then the solution still represents the tachyon vacuum, although in a non-universal form. So non trivial RG fixed points are described when $\frac{1}{K+\phi_{u}}$ is singular but $\frac{B}{K+\phi_{u}} u \partial_{u} \phi_{u}$ is finite (since the solution itself must be finite). Remarkably, the trace of the matter part of this last quantity turns out to coincide with the shift in the partition function from the UV to the IR on the canonical cylinder of width 1.

$$
\operatorname{Tr}\left[\frac{1}{K+\phi_{u}} u \partial_{u} \phi_{u}\right]=\left(\lim _{u \rightarrow 0}-\lim _{u \rightarrow \infty}\right)\left\langle e^{-\int_{0}^{1} d s \phi_{u}(s)}\right\rangle_{C_{1}} .
$$

Thanks to this important identity, the closed string overlap, [43], is shown to coincide with the shift in the disk-one-point function of an on-shell closed string. Similar considerations apply for the on-shell value of the action. We show that the energy is independent of the gauge parameter $u$ and we write down a closed regular expression for it. Due to the essentially more involved structure wrt the closed string overlap, we have postponed its explicit calculation.

The paper is organized as follows. In section 2 we show that all we are going to discuss is already hinted at by the universal tachyon vacuum solutions, in a very simple way: this is expected since the tachyon vacuum is the simplest IR fixed point that one gets by integrating on the boundary of the worldsheet a zero momentum tachyon. We take the time to make some simple yet important observation about the Erler-Schnabl solution, [4]. Thanks to the structure of the solution, it is very easy to identify the world-sheet RG flow and to naturally associate the UV fixed point with the identity string field and the IR with the sliver, where a zero momentum tachyon is integrated along the boundary. We show how the integration over the Schwinger parameter, upon evaluation of gauge invariant quantities, reduces to the shift between the UV and IR of the corresponding observables.

In section 3 we algebraically build the solution, by writing a pure gauge ansatz, inspired by the reparametrizations of the Erler-Schnabl tachyon vacuum. To do so we minimally extend the $K, B, c$ algebra to contain the identity-based insertion of a relevant operator and its BRST transform. No further extension is needed to close the multiplicative differential algebra. Inside this algebra we point out how to build wedge-like surfaces, where the relevant operator is exp-integrated along the boundary. The solution we write down is somehow similar to the Erler-Schnabl tachyon vacuum in the sense that it is a continuous superposition of wedge states, however such wedges have deformed boundary conditions given by exp-integrating the relevant field on the boundary. Given this crucial difference wrt universal tachyon vacuum solutions, we nonetheless show that the solution is still 'very close' to just be the same tachyon vacuum, expressed in a non-universal way. We then state the crucial conditions under which we can have a solution which is different from the tachyon vacuum.

In section 4 we determine some sufficient conditions which allow to relate the abovementioned conditions to the IR limit of the partition function along the RG flow: the disk 
partition function should be finite and not vanishing in the IR. As a matter of fact, this crucial requirement has been already pointed out by Ellwood, [39], in order to guarantee the existence of a finite characteristic projector. We then show how the shift in the partition function is encoded inside the solution. A consequence of this, section 5, is the fact that the Ellwood invariants of the solution correctly capture the shift in the closed string one-point function between the UV BCFT and the IR one. In section 6 we write down the energy functional of the solution, and we discuss it to some extent.

In section 7 we give concrete examples of solutions describing well known RG-flows: lower dimensional branes in non compact and compact dimensions. We end up with some conclusions and open problems.

A few appendices contain additional material: the construction of the solution using the homotopy field at the tachyon vacuum (Appendix A), the explicit proof that the two non real forms of the solution have the same energy and that the energy can indeed be written as the integration of an explicitly real string field (Appendix B), the generalization of the solution to the (cubic) superstring case (Appendix C, with the left unanswered interesting question on wheatear cubic superstring field theory contains solutions which are not expected from string theory, such as codimension $1 \mathrm{D}$-branes in the $\mathrm{GSO}(+)$ sector). Some explicit computations for the simplest relevant deformation based on $\int: X^{2}$ : are reviewed and presented in Appendix D. Finally, Appendix E is the derivation of equation (3.27).

\section{Reparametrizations of the Erler-Schnabl Tachyon Vacuum}

The quickest way to arrive at the 'relevant' solutions is a very simple yet illuminating observation about the recent solution derived by Erler and Schnabl, describing the tachyon vacuum. The solution, [4], is given by

$$
\psi_{0}=\frac{1}{1+K} c(1+K) B c=c-\frac{1}{1+K} B c \partial c
$$

and can be formally obtained with a singular gauge transformation of the perturbative vacuum

$$
\begin{aligned}
\psi_{0} & =U_{0} Q U_{0}^{-1} \\
U_{0} & =1-\frac{1}{1+K} B c \\
U_{0}^{-1} & =1+\frac{1}{K} B c .
\end{aligned}
$$

A trivial infinite class of gauge equivalent solutions can be obtained by a simple scale transformation (which, on the cylinder, is a midpoint preserving reparametrization)

$$
z \rightarrow \frac{z}{u}
$$


under which the $K B c$ algebra is mapped to an isomorphic (i.e. equivalent) representation

$$
\begin{aligned}
c & \rightarrow u c \\
(B, K) & \rightarrow \frac{1}{u}(B, K) .
\end{aligned}
$$

The new solutions are thus generated by the gauge transformations

$$
\begin{aligned}
U_{u} & =1-\frac{u}{u+K} B c \\
U_{u}^{-1} & =1+\frac{u}{K} B c,
\end{aligned}
$$

giving the reparametrizations of the tachyon vacuum

$$
\psi_{u}=\frac{u}{u+K} c(u+K) B c=u c-\frac{u}{u+K} B c \partial c .
$$

Since reparametrizations do not change the bpz norm (and consequently the gauge invariant action and closed string overlap) we have

$$
\begin{aligned}
\left\langle\psi^{(u)}, Q \psi^{(u)}\right\rangle & =\left\langle\psi^{(u=1)}, Q \psi^{(u=1)}\right\rangle \\
\left\langle V_{c}, \psi^{(u)}\right\rangle & =\left\langle V_{c}, \psi^{(u=1)}\right\rangle
\end{aligned}
$$

as can also be explicitly checked by rescaling the involved correlators on the cylinders.

A naive extrapolation would erroneously lead us to believe the above result to hold for any value of $u$. However we must set $u>0$ in order to ensure that, under the scaling, all wedge states $\left(e^{-t K} \rightarrow e^{-\frac{t}{u} K}\right)$ remain of finite positive width. A negative value of $u$ would result in inverse wedge states (wedge states with negative width), which are ill-defined objects $\left(e^{t K}\right)$. One must also note that blindly setting $u=0$ is not the same as taking the limit $u \rightarrow 0^{+}$. In the former case the gauge transformations become the identity and the perturbative vacuum (D25 brane) remains trivially untouched; but by taking the $u \rightarrow 0^{+}$ limit a phantom piece is switched on

$$
\lim _{u \rightarrow 0^{+}} \frac{u}{u+K}=\text { sliver }
$$

and the corresponding solution is still the tachyon vacuum (although singularly reparametrized). To summarize, we have

$$
\begin{aligned}
& u>0 \rightarrow \text { Tachyon Vacuum } \\
& u \equiv 0 \rightarrow \text { D25-brane } \\
& u<0 \rightarrow \text { Ill-defined solution. }
\end{aligned}
$$

The above observation has a simple physical interpretation in terms of the tachyon potential. The gauge parameter $u$ is like an initial displacement applied to the system at the perturbative vacuum in order to reach another stationary point. For $u>0$ the displacement is in the right direction to reach the tachyon vacuum. For $u=0$ there is no displacement at all, so the solution will stay at the perturbative vacuum. For $u<0$ the 
displacement is in the wrong direction, and the solution falls in the unbounded region of the potential, becoming singular.

This target-space perspective can be brought (and made more precise) to the worldsheet. The trivial gauge parameter $u$ can be interpreted as the value during the RG flow of a zero momentum tachyon integrated along the boundary. To see this, the crucial quantity to analyze is 1

$$
\operatorname{Tr}\left[\frac{u}{u+K}\right]=\int_{0}^{\infty} d T u\left\langle e^{-u T}\right\rangle_{C_{T}}=\int_{0}^{\infty} d T u\left\langle e^{-\int_{0}^{T} d s u}\right\rangle_{C_{T}},
$$

rescaling to a canonical cylinder of width 1

$$
\begin{aligned}
\operatorname{Tr}\left[\frac{u}{u+K}\right] & =\int_{0}^{\infty} d T u\left\langle e^{-\int_{0}^{1} d s(T u)}\right\rangle_{C_{1}} \\
& =\int_{0}^{\infty} d T\left(-\partial_{T}\right)\left\langle e^{-\int_{0}^{1} d s(T u)}\right\rangle_{C_{1}} \\
& =\left(\lim _{u \rightarrow 0^{+}}-\lim _{u \rightarrow \infty}\right)\left\langle e^{-\int_{0}^{2 \pi} \frac{d \theta}{2 \pi} u}\right\rangle_{D i s k}=Z_{u=0}-Z_{u=\infty},
\end{aligned}
$$

where by

$$
Z(u) \equiv\left\langle e^{-\int_{0}^{2 \pi} \frac{d \theta}{2 \pi} u}\right\rangle_{D i s k}=e^{-u},
$$

we denote the disk partition function obtained by integrating a zero momentum tachyon on the disk (or cylinder) boundary.

We see that the quantity we have computed is just the shift in the partition function between the perturbative vacuum (UV fixed point) and the tachyon vacuum (IR fixed point), where the partition function identically vanishes due to the infinite suppression given by the zero momentum tachyon which is sent to $\infty$ during the RG flow.

Needless to say this is the way the tachyon condenses in Boundary String Field Theory, but the reader should not be misled by this: in BSFT the zero momentum tachyon field extremizes the action only when $u=0$ (no deformation present) or when $u=\infty$ (endpoint of the RG flow). In cubic OSFT -more precisely, in the Erler-Schnabl description of the tachyon vacuum- $u$ is a trivial gauge parameter: the shape of the tachyon field is meaningless (it is not gauge invariant) but the gauge invariant observables exactly capture the entire jump between the UV and the IR of the worldsheet RG flow, independently of the initial value of $u$. In other words, the Erler-Schnabl solution (with its reparametrizations) is just what is needed to explicitly 'see' the RG flow at work on the world-sheet and, in this sense, it can be considered a 'BSFT'-like solution. It would be interesting to generalize this discussion to other universal solutions (for example to the original Schnabl solution, where a simple reparametrization seems to give much less interesting structure).

Another important remark is about the role of the star algebra projectors in this mechanism: the integration over the Schwinger parameter reduces to the difference between

\footnotetext{
${ }^{1} \mathrm{~A}$ BRST invariant insertion of $Y(z)=\frac{1}{2} \partial^{2} c \partial c c(z)$ is understood to saturate the ghost number. The reader can easily verify that this simple trace captures all the essential features of the closed string overlap, in particular the shift from the UV to the IR.
} 
the norm of the identity string field (which gives the UV fixed point) and that of the sliver with a relevant boundary integration (which gives the IR fixed point). It is really the sliver limit that drives the RG flow all the way to the IR. This is yet another nice property of sliver-like projectors inside the star algebra: rescaling to finite width a very large wedge state, dressed with a relevant boundary exp-integration, brings the relevant deformation to its IR fixed point, in the sliver limit. ${ }^{2}$

These considerations suggest that a lump solution can be obtained by replacing the gauge parameter $u$ with a properly chosen tachyon profile $\phi(x)$. Heuristically, from a target space perspective, $\phi(x)=0$ will correspond to regions in the transverse space where the theory is at the perturbative vacuum, while for $\phi(x)>0$ the theory will be at the tachyon vacuum. More precisely, in the far infrared of the worldsheet, the minima of $\phi(x)$ will correspond to the regions where all the energy will be stored, [33], that is the location of the lower dimensional branes. However, since $X$ is a quantum field and not a number, the height of the minima of $\phi(x)$ should be very carefully balanced in order to have a well defined (and non trivial) IR fixed point. We also learn that a 'localized' lump solution (i.e. a tachyon with a localized dependence on the $X$-zero mode) is not a requirement as, already for the tachyon vacuum, the tachyon VEV can be freely changed (and even pushed to infinity) by a scale transformation in the arctan frame.

\section{The solution: general structure}

\subsection{Adding a relevant field to the $K, B, c$ algebra}

The solution lives in a minimal extension of the universal $K, B, c$ subalgebra, which can be defined in the sliver frame (obtained by mapping the UHP to an infinite cylinder $C_{2}$ of circumference 2 , by the sliver map $\left.f(z)=\frac{2}{\pi} \arctan z\right) \mathrm{as}^{3}$

$$
\begin{aligned}
K & =\frac{\pi}{2} K_{1}^{L}|I\rangle \\
B & =\frac{\pi}{2} B_{1}^{L}|I\rangle \\
c & =c\left(\frac{1}{2}\right)|I\rangle .
\end{aligned}
$$

They obey the well known star-product relations

$$
\begin{aligned}
\{B, c\} & =1 \\
{[K, B] } & =0 \\
{[K, c] } & \equiv \partial c \\
\{B, \partial c\} & =0,
\end{aligned}
$$

as well as the differential relations

$$
\begin{aligned}
Q B & =K \\
Q c & =c K c=c \partial c .
\end{aligned}
$$

\footnotetext{
${ }^{2}$ In fact this was already noted by Rastelli, Sen and Zwiebach, 40], during the 'VSFT' era. A consequence of this is that the deformed sliver will have the boundary conditions of the IR BCFT at its midpoint, [39].

${ }^{3}$ Local insertions of fields are defined on $C_{2}$ so that, for primaries, $\chi_{h}^{\left(C_{2}\right)}(1 / 2)=\pi^{h} \chi_{h}^{(U H P)}(1)$.
} 
A relevant matter operator $\phi$ (not necessarily primary) is now added. As for $K, B, c$ we introduce it as an identity-based insertion in the sliver frame.

$$
\phi=\phi\left(\frac{1}{2}\right)|I\rangle
$$

The new simple $*$-product relations are given by

$$
\begin{aligned}
{[c, \phi] } & =0 \\
{[B, \phi] } & =0 \\
{[K, \phi] } & \equiv \partial \phi,
\end{aligned}
$$

as well as

$$
\begin{aligned}
{[c,[K, \phi]] } & =[c, \partial \phi]=0 \\
{[\phi,[K, c]] } & =[\phi, \partial c]=0 \\
{[B,[K, \phi]] } & =[B, \partial \phi]=0 .
\end{aligned}
$$

The BRST charge acts on $\phi$ in the following way

$$
Q \phi=c \partial \phi+\partial c \delta \phi
$$

When $\phi$ is a primary of weight $h$ we simply have

$$
Q \phi=c \partial \phi+h \partial c \phi
$$

If $\phi$ is not primary, then $\phi$ needs not be proportional to $\delta \phi$, which can be explicitly computed from the BRST variation. In order to avoid the explicit appearance of $\partial \phi$ in the solution we will write down below, it is useful to introduce the insertion $\phi$ together with a $c$ ghost in front. This gives

$$
Q(c \phi)=c \partial c(\phi-\delta \phi)
$$

If $\phi$ is a primary of dimension $h$ we then have

$$
Q(c \phi)=c \partial c(1-h) \phi
$$

which correctly vanishes when $\phi$ is marginal.

An important assumption we will make about the matter insertion $\phi$ is that its OPE should be such as to make ${ }^{4}$

$$
(c \phi)^{2}=(c \phi)(c \delta \phi)=(c \delta \phi)(c \phi)=0
$$

\footnotetext{
${ }^{4}$ The assumption is actually more general. In order to define deformed wedge states without the need of a renormalization of contact term divergences, see later, we need the non-local operator $e^{-\int_{a}^{b} d s \phi(s)}$ to be finite by itself. This (at least in conformal perturbation theory) is an assumption about the finiteness of the integrated $N$-point functions $\int d s_{1} \ldots \int d s_{N}\left\langle\phi\left(s_{1}\right) \ldots \phi\left(s_{N}\right)\right\rangle^{\left(B C F T_{0}\right)}$, which is satisfied in all the explicit examples we consider in the present paper.
} 
In the case where $\phi=\phi^{(h)}$ is a boundary primary field of weight $h$, with a singular OPE on the UHP of the kind

$$
\phi(x)^{(h)} \phi(0)^{(h)} \sim \frac{a}{|x|^{2 h}}, \quad x \in \mathfrak{R}
$$

this implies that

$$
h_{\phi}<\frac{1}{2}
$$

In particular, differently from the marginal case, here (mild) singular collisions between the $\phi$ 's are allowed. ${ }^{5}$

Inside this algebra one can build wedge-like surfaces, where the matter field $\phi$ is integrated along the boundary. To do this one simply adds $\phi$ to the strip-generator $K$

$$
K \rightarrow K+\phi
$$

and exponentiate it, see also [38]. That is, a deformed wedge state of width $T$ is given by

$$
|T, \phi\rangle=e^{-T(K+\phi)} .
$$

The overlap with a Fock space test state $\chi=\chi(0)|0\rangle$ is given by

$$
\langle\chi \| T, \phi\rangle=\left\langle f \circ \chi(0) e^{-\int_{\frac{1}{2}}^{T+\frac{1}{2}} d s \phi(s)}\right\rangle_{C_{T+1}},
$$

where

$$
f(z)=\frac{2}{\pi} \arctan z
$$

and we have used the fact that, inside the path integral, we can represent (see Appendix E or [38] for a derivation)

$$
\left\langle[\ldots] e^{-T(K+\phi)}[\ldots]\right\rangle=\left\langle[\ldots] e^{-T K} e^{-\int_{x}^{x+T} d s \phi(s)}[\ldots]\right\rangle .
$$

In other words, just as $e^{-T K}$ generates a strip of worldsheet with unmodified boundary conditions, $e^{-T(K+\phi)}$ generates the same strip but it also inserts in the path integral $e^{-\int_{x}^{x+T} d s \phi(s)}$, which gives modified (non conformal unless $\phi$ is exactly marginal) boundary conditions. This operation requires a regularization and a consequent renormalization in the case of marginal $\phi$ with singular OPE, but it is well defined when $\phi$ is relevant enough, i.e. it satisfies (3.21), see [36] for an explicit example of how the divergences in the $N$-point functions of $\phi$ gives rise to integrable singularities, with an overall finite result. We do not attempt here to find a regularization procedure to treat all kind of relevant fields, but we will stick to (3.21).

\footnotetext{
${ }^{5}$ It is clear that one can also consider linear combinations of primaries with global regular OPE, with the only restriction that they should be relevant $(0 \leq h<1)$, this could be useful, for example, to get non-marginal time dependent solutions.
} 
It is important to be aware that, unless $\phi$ is BRST closed and (since it is a matter field) proportional to the identity operator (that is, a pure number) the deformed wedge state is not BRST closed

$$
Q|T, \phi\rangle=Q e^{-T(K+\phi)}=\int_{0}^{T} d t e^{-t(K+\phi)}(-Q \phi) e^{-(T-t)(K+\phi)} \neq 0 .
$$

This is also true when $\phi=j$ is marginal, however in that case the above integral can be recast in

$$
Q\left(e^{-T(K+j)}\right)=-\left[c j, e^{-T(K+j)}\right],
$$

which implies that the BRST variation of the deformed wedge is just a 'boundary' contribution: this is indeed what happens by deforming the boundary conditions while preserving conformal invariance, 23. In the case $\phi$ is relevant, on the other hand, the BRST violation has also a term which cannot be pushed to the extremes of the wedge

$$
Q\left(e^{-T(K+\phi)}\right)=-\left[c \phi, e^{-T(K+\phi)}\right]+\int_{0}^{T} d t e^{-t(K+\phi)}[K, c](\phi-\delta \phi) e^{-(T-t)(K+\phi)} .
$$

This is the reflection of the fact that the boundary conditions given by inserting $e^{-\int \phi}$ in the path integral are not conformal. ${ }^{6}$

The possibility of building wedge states with deformed (non conformal) boundary conditions is the basic property thanks to which a boundary RG flow can be engineered inside a minimal extension of the $K, B, c$ algebra: by gluing together the deformed wedge states, one generates surfaces where a relevant operator is integrated along the boundary. The original scale invariance of the world sheet path integral will now generate an RG flow which will drive the system from the perturbative vacuum, where our OSFT is defined, to a new conformal fixed point.

\subsection{Solution from pure gauge ansatz}

Two simple non real solutions can be constructed, which are the 'reality'-conjugate of one another (they can be obtained from one another by reading them in the opposite sense). Focusing on the 'left' solutions (as in the previous section) we write down the following gauge transformation

$$
\begin{aligned}
U_{\phi} & =1-\frac{1}{K+\phi} \phi B c \\
U_{\phi}^{-1} & =1+\frac{1}{K} \phi B c,
\end{aligned}
$$

the collisions between the $\phi$ 's in the denominator and in the numerator of $U$ do not give rise to divergences if (3.21) holds, i.e. if $\phi(z) \phi(0)=o\left(|z|^{-1}\right)$.

Notice that these gauge transformations are obtained by substituting the $u$ of the previous section with the relevant field $\phi$. In doing this there are ambiguities in the position

\footnotetext{
${ }^{6}$ In the trivial case where $\phi$ is a non zero constant the two pieces in the rhs of the above equation identically cancel, however this does not mean that the boundary conditions are conformal.
} 
of $\phi$ and $K$ (the two operators do not commute). In this particular order both $U$ and $U^{-1}$ are relatively simple, and they do not give rise to $1 / K$ factors in the solution. In Appendix A we show how these gauge transformations can be obtained from a singular gauge transformation of the tachyon vacuum, following the procedure suggested by Ellwood, [39]. Here we just stick to this form, leaving open the interesting question about the existence of other kinds of gauge transformations that could result in more general solutions. We call the relevant field $\phi$, to which the solution is associated, the seed.

More importantly, notice that, because of the explicit $1 / K, U^{-1}$ is certainly singular (except for the trivial case $\phi \equiv 0$ ), while $U$ can be regular or singular according to the behavior of the string field $\frac{1}{K+\phi}$. As we will show in a while, if this string field turns out to be regular, then the solution will just be a non universal way of writing down the tachyon vacuum. In fact, see next subsection, only a singular $\frac{1}{K+\phi}$ can give rise to a new solution. Anticipating the results of the next section, this is related to the fact that the IR partition function of the relevant boundary deformation given by the integration of $\phi$ should be finite and not vanishing in the IR. A vanishing partition function in the IR would mean that the IR fixed point that has been reached is still the tachyon vacuum and not a lower dimensional brane (or a new genuinely propagating BCFT).

Using the BRST variation

$$
Q(c \phi)=(\phi-\delta \phi) c[K, c]=(\phi-\delta \phi) c \partial c
$$

we get

$$
\begin{aligned}
\psi_{\phi}=U_{\phi} Q U_{\phi}^{-1} & =\left(1-\frac{1}{K+\phi} \phi B c\right) Q\left(1+\frac{1}{K} \phi B c\right) \\
& =\left(1-\frac{1}{K+\phi} \phi B c\right)\left(c \phi-\frac{B}{K}(\phi-\delta \phi) c \partial c\right) \\
& =c \phi-\frac{1}{K+\phi}(K+\phi-\phi) \frac{1}{K}(\phi-\delta \phi) B c \partial c \\
& =c \phi-\frac{1}{K+\phi}(\phi-\delta \phi) B c \partial c .
\end{aligned}
$$

Notice that there is a naked identity-based piece in the solution. However, because $c \phi$ has negative net scaling dimension (since $\phi$ is relevant), the typical ambiguities related to identity-based string fields are here avoided. ${ }^{7}$

It is important to observe that, in the case where $\phi$ is a marginal operator (with trivial $\mathrm{OPE}$, so that no renormalization of the boundary integral is needed) only the identity piece $c \phi$ survive $(\phi-\delta \phi=0)$. This is a trivial solution of the equation of motion but, due to its vanishing scaling dimension, the associated observables (energy and closed string overlap) are undefined (they depend on the way the limit $\epsilon \rightarrow 0$ is taken). So, from the point of view of identity-like singularities, our solution is only acceptable for a truly relevant $\phi$.

\footnotetext{
${ }^{7}$ As explained in [4], this can be made more precise by understanding the seed $\phi$ as
}

$$
\phi=\lim _{\epsilon \rightarrow 0} \phi \Omega^{\epsilon}, \quad \Omega=e^{-K} .
$$


Remember also that this solution describes a universal tachyon vacuum by simply choosing a constant, positive $\phi$ (the zero momentum tachyon).

As anticipated two (non real) solutions can be constructed

$$
\begin{aligned}
U_{L} & =1-\frac{1}{K+\phi} \phi B c \\
\psi_{L} & =U_{L} Q U_{L}^{-1}=c \phi-\frac{1}{K+\phi}(\phi-\delta \phi) B c \partial c \\
\cdot & \\
U_{R} & =1-c B \phi \frac{1}{K+\phi} \\
\psi_{R} & =U_{R}^{-1} Q U_{R}=c \phi-c \partial c B(\phi-\delta \phi) \frac{1}{K+\phi}=\psi_{L}^{\ddagger},
\end{aligned}
$$

where $\ddagger$ is the reality-conjugation, 42

$$
\ddagger \equiv \mathrm{bpz}^{-1} \circ \mathrm{hc} .
$$

Since the two solutions are written in pure gauge form, they will, at least formally, automatically solve the equation of motion. With some manipulations in the $K, B, c ; \phi$ algebra one can show that ${ }^{8}$

$$
Q \psi_{L}=\left(c \phi-\frac{1}{K+\phi}(\phi-\delta \phi) \partial c\right) \frac{1}{K+\phi}(\phi-\delta \phi) B c \partial c=-\psi_{L}^{2}
$$

and accordingly

$$
\begin{aligned}
Q \psi_{R} & =c \partial c B(\phi-\delta \phi) \frac{1}{K+\phi}\left[c \phi+\partial c(\phi-\delta \phi) \frac{1}{K+\phi}\right] \\
& =c \partial c B(\phi-\delta \phi) \frac{1}{K+\phi} \psi_{R}=-\psi_{R}^{2} .
\end{aligned}
$$

Notice that, differently from the tachyon vacuum solution, two Schwinger parameters are needed for $Q \psi$. Due to the particular form of the solutions, and to the commutation relations of the $K, B, c, \phi$ algebra

$$
\begin{aligned}
\{B, \partial c\} & =0 \\
{[B, K]=[B, \phi] } & =[c, \phi]=[\partial c, \phi]=0,
\end{aligned}
$$

it is easy to prove that the two solutions share the same energy

$$
\operatorname{tr}\left[\psi_{R} \psi_{R} \psi_{R}\right]=\operatorname{tr}\left[\psi_{L} \psi_{L} \psi_{L}\right]
$$

\footnotetext{
${ }^{8}$ Here we are assuming that it is possible to define the string field $\left[\frac{1}{K+\phi}(\phi-\delta \phi)\right]$ such that$$
(K+\phi)\left[\frac{1}{K+\phi}(\phi-\delta \phi)\right]=\phi-\delta \phi
$$

See [55] and [56, 57, where this problem is discussed, using two different attitudes. 
and the same closed string overlap

$$
\operatorname{tr}\left[V_{c} \psi_{R}\right]=\operatorname{tr}\left[V_{c} \psi_{L}\right]
$$

In Appendix B an explicit real form of the energy is given. With some algebra one can also check that the two solution are related by a very simple gauge transformation in the $K, B, c, \phi$ algebra

$$
\psi_{L}=\frac{1}{K+\phi}\left(Q+\psi_{R}\right)(K+\phi)
$$

This allows to write (quite formally) a real solution as

$$
\psi=\psi^{\ddagger}=\frac{1}{\sqrt{K+\phi}}\left(Q+\psi_{R}\right) \sqrt{K+\phi}=\sqrt{K+\phi}\left(Q+\psi_{L}\right) \frac{1}{\sqrt{K+\phi}} .
$$

\subsection{Is this different from the Tachyon Vacuum?}

It is instructive to write down the kinetic operator around this solution to see if it is possible to trivialize it. With some manipulation, using the $K, B, c, \phi$ algebra it is possible to show that

$$
\mathcal{Q}_{\psi_{\phi}} \frac{B}{K+\phi}=Q \frac{B}{K+\phi}+\left\{\psi_{\phi}, \frac{B}{K+\phi}\right\}=1
$$

So, unless the homotopy-field $\frac{B}{K+\phi}$ is ill-defined (as it is the case for $\frac{B}{K}$ and the original $Q$ ), the solution has trivial cohomology, which is the defining property of the tachyon vacuum [41]. On the other hand, in order for the solution to be finite, the quantity $\frac{B}{K+\phi}(\phi-\delta \phi)$ should be itself finite (at least in the 'weak' sense that it should give rise to well defined observables, and finite overlaps with Fock-space states). In full generality we thus have a new nontrivial solution if

- $\frac{1}{K+\phi}$ is divergent

- $\frac{B}{K+\phi}(\phi-\delta \phi)$ is finite.

We will now give some sufficient conditions for the seed $\phi$ in order to comply with the above requirement.

\section{A class of nontrivial solutions}

We introduce here a general class of seeds $\phi$ that give rise to Fock-space-finite non trivial solutions. We will later give explicit examples of seeds in this class. Let $u$ be a parametrization of the world-sheet RG flow represented by integrating a relevant vertex operator $\phi_{u}$ on the boundary. We take $u$ to span the positive real axis with $u=0$ corresponding to the UV (no deformation present) and $u=\infty$ to the IR. In particular we have

$$
\phi_{u=0}=0 .
$$


The first important condition is that $u$ should linearly increase under scaling in the cylinder coordinate frame. That is

$$
f_{t}(z)=\frac{z}{t}
$$

should imply

$$
f_{t} \circ \phi_{u}(z)=\frac{1}{t} \phi_{t u}\left(\frac{z}{t}\right)
$$

This condition actually states that $u$ can be changed to any positive value by a scale transformation. Thus $u$-dependence of the solution will be a gauge artifact.

The second, related, condition is about the BRST variation of $c \phi_{u}$. We will require that

$$
Q\left(c \phi_{u}\right)=c \partial c\left(\phi_{u}-\delta \phi_{u}\right)=c \partial c u \partial_{u} \phi_{u}
$$

Notice that the above conditions are trivially satisfied for the seed giving rise to the tachyon vacuum, where one just chooses

$$
\phi_{u}=u>0
$$

The third condition is related to our aim to avoid flowing to the tachyon vacuum in the IR, that is to the requirement that at the IR fixed point some perturbative open string dynamics is left. In order for this to happen the partition function on the canonical cylinder of width 1 (or equivalently on the unit disk) should be finite and not vanishing, at the end of the RG-flow

$$
\lim _{u \rightarrow \infty}\left\langle e^{-\int_{0}^{1} d s \phi_{u}(s)}\right\rangle_{C_{1}}=\text { finite. }
$$

The importance of this condition, which is usually ignored in the literature on worldsheet RG flows, has been originally stressed by Ellwood, [39], whose intuition is here made concrete.

Now we want to show that these conditions are just enough to guarantee that $\frac{1}{K+\phi_{u}}$ is divergent while $\operatorname{Tr}\left[\frac{1}{K+\phi_{u}}\left(\phi_{u}-\delta \phi_{u}\right)\right]$ is well defined. Let us first consider

$$
\operatorname{Tr}\left[\frac{1}{K+\phi_{u}}\right]=\int_{0}^{\infty} d T\left\langle e^{-\int_{0}^{T} d s \phi_{u}(s)}\right\rangle_{C_{T}}=(*),
$$

by a scale transformation, using (4.2) we get

$$
(*)=\int_{0}^{\infty} d T\left\langle e^{-\int_{0}^{1} d s \phi_{T u}(s)}\right\rangle_{C_{1}}=\int_{0}^{\infty} d T g(T u),
$$

where we denoted

$$
g(v) \equiv \operatorname{Tr}\left[e^{-\left(K+\phi_{v}\right)}\right]=\left\langle e^{-\int_{0}^{1} d s \phi_{v}(s)}\right\rangle_{C_{1}},
$$

the partition function on the canonical cylinder obtained by integrating $\phi_{v}$ on the boundary. This partition function is in turn identified with the exp of boundary entropy and from the 
$g$-theorem/conjecture, [32], we know that $g(v)$ is a monotonically decreasing (and positive) function of $v$. But because of condition (4.5) $g(u T)$ will asymptote to a finite non vanishing positive value for $T \rightarrow \infty$. Thus the integral diverges. This shows that the string field $\frac{1}{K+\phi_{u}}$ is singular as required for the solution to be different from the tachyon vacuum. Let us now analyze the trace of the quantity entering the solution, that is

$$
\operatorname{Tr}\left[\frac{1}{K+\phi_{u}}\left(\phi_{u}-\delta \phi_{u}\right)\right]=\operatorname{Tr}\left[\frac{1}{K+\phi_{u}} u \partial_{u} \phi_{u}\right]=\int_{0}^{\infty} d \operatorname{Tr}\left[e^{-T\left(K+\phi_{u}\right)} u \partial_{u} \phi_{u}\right]
$$

where condition (4.3) has been used. Recalling the derivative of a non-commutative exponential

$$
d\left(e^{M}\right)=\int_{0}^{1} d s e^{s M}(d M) e^{(1-s) M}
$$

and simplifying it inside a trace

$$
\operatorname{Tr}\left[d\left(e^{M}\right)\right]=\operatorname{Tr}\left[\int_{0}^{1} d s e^{s M}(d M) e^{(1-s) M}\right]=\int_{0}^{1} d s \operatorname{Tr}\left[(d M) e^{(1-s+s) M}\right]=\operatorname{Tr}\left[(d M) e^{M}\right],
$$

we have

$$
\begin{aligned}
u \int_{0}^{\infty} d \operatorname{Tr}\left[\partial_{u} \phi_{u} e^{-t\left(K+\phi_{u}\right)}\right] & =-\int_{0}^{\infty} d t \frac{u}{t} \partial_{u} \operatorname{Tr}\left[e^{-t\left(K+\phi_{u}\right)}\right] \\
& =-\int_{0}^{\infty} d t \frac{u}{t} \partial_{u}\left\langle e^{-\int_{0}^{T} \phi_{u}(s) d s}\right\rangle_{C_{T}} \\
& =-\int_{0}^{\infty} d t \frac{u}{t} \partial_{u} g(t u)=-\int_{0}^{\infty} d x \partial_{x} g(x)
\end{aligned}
$$

To summarize, we have the very remarkable property

$$
\operatorname{Tr}\left[\frac{1}{K+\phi_{u}}\left(\phi_{u}-\delta \phi_{u}\right)\right]=g(0)-g(\infty)=Z^{U V}-Z^{I R}
$$

Not only is this quantity perfectly well defined but it also precisely agrees with the shift in the open string partition function from the UV to the IR! $!^{9}$ Notice that we have used the conditions $4.2,4.3,4.5$ ) to arrive at this remarkable result. Whether these conditions are also necessary, or can be modified in some way, is an interesting problem we leave for further investigation. It turns out, see [55, 56], that finiteness of $\operatorname{Tr}\left[\frac{1}{K+\phi_{u}}\left(\phi_{u}-\delta \phi_{u}\right)\right]$ is enough to guarantee finiteness of $\psi_{L}$ inside observables and against Fock space states.

\section{Closed String overlap}

The simplest gauge invariant quantity that can be explicitly computed for the class of solutions defined in the previous section is the closed string overlap, i.e. the overlap between the solution and an on-shell closed string state inserted at the midpoint of the identity

\footnotetext{
${ }^{9}$ If we work in flat non compact space, then $g(0)$ will obviously diverge because of the infinite volume, we do not consider this to be a sign of 'non regularity', since it is just the reflection that $X$ has a non compact zero mode.
} 
string field. According to Ellwood, [43], this quantity should be equal to the shift in the closed string one-point function between the new BCFT represented by the new solution and the reference BCFT represented by the perturbative vacuum. In full generality, if $\psi_{1}$ represents the new $B C F T_{1}$, expressing everything on a canonical cylinder of width 1, we expect to find

$$
\operatorname{Tr}\left[V_{c} \psi_{1}\right]=\left\langle V_{c}(i \infty) c(0)\right\rangle_{C_{1}}^{\left(B C F T_{0}\right)}-\left\langle V_{c}(i \infty) c(0)\right\rangle_{C_{1}}^{\left(B C F T_{1}\right)}
$$

In the case $B C F T_{1}$ is the IR fixed point of a relevant boundary interaction, the correlator in $B C F T_{1}$ can be seen as a correlator in $B C F T_{0}$ with an exp-boundary integral, responsible for the change in boundary conditions from $B C F T_{0}$ to $B C F T_{1}$.

$$
\left\langle V_{c}(i \infty) c(0)\right\rangle_{C_{1}}^{\left(B C F T_{1}\right)}=\lim _{v \rightarrow v^{*}}\left\langle V_{c}(i \infty) c(0) e^{-v \int_{0}^{1} d t \theta(t)}\right\rangle_{C_{1}}^{\left(B C F T_{0}\right)},
$$

where $v^{*}$ is the IR fixed point in coupling space. Therefore

$$
\begin{aligned}
\operatorname{Tr}\left[V_{c} \psi_{1}\right] & =\left\langle V_{c}(i \infty) c(0)\left(1-\lim _{v \rightarrow v^{*}} e^{-v \int_{0}^{1} d t \theta(t)}\right)\right\rangle_{C_{1}}^{\left(B C F T_{0}\right)} \\
& =\operatorname{Tr}\left[V_{c} c\left(\Omega-\lim _{v \rightarrow v^{*}} \tilde{\Omega}_{v}\right)\right]
\end{aligned}
$$

where we have used the boundary-changed vacuum $\tilde{\Omega}_{v}$

$$
\begin{aligned}
\Omega & =e^{-K} \\
\tilde{\Omega}_{v} & =e^{-K-v \theta} .
\end{aligned}
$$

Our solution is given by

$$
\psi_{u}=c \phi_{u}-\frac{1}{K+\phi_{u}}\left(\phi_{u}-\delta \phi_{u}\right) B c \partial c=c \phi_{u}-\frac{1}{K+\phi_{u}} u \partial_{u} \phi_{u} B c \partial c,
$$

where we have already chosen a seed $\phi_{u}$ such that (4.3) is satisfied. The identity piece in the solution does not contribute, thanks to the globally negative scaling dimension (the seed is relevant). In particular, using the scaling property (4.2), we have

$$
\operatorname{Tr}\left[V_{c} c \phi_{u}\right]=\lim _{\epsilon \rightarrow 0} \operatorname{Tr}\left[V_{c} c \phi_{u} \Omega^{\epsilon}\right]=\lim _{\epsilon \rightarrow 0} \operatorname{Tr}\left[V_{c} c \phi_{\epsilon u} \Omega^{1}\right]=0,
$$

because

$$
\phi_{u=0} \equiv 0
$$

since no deformation is present in the UV. So only the second term of the solution enters the closed string overlap. We have thus to compute

$$
\begin{aligned}
\operatorname{Tr}\left[V_{c} \psi_{u}\right] & =-\operatorname{Tr}\left[V_{c} \frac{1}{K+\phi_{u}} u \partial_{u} \phi_{u} B c \partial c\right] \\
& =-\int_{0}^{\infty} d T\left\langle V_{c}(i \infty) B c \partial c(0) u \partial_{u} \phi_{u}(0) e^{-\int_{0}^{T} d s \phi_{u}(s)}\right\rangle_{C_{T}}
\end{aligned}
$$


rescaling $C_{T} \rightarrow C_{1}$ (the closed string is scale invariant since it is on shell) and changing variable $y=T u$ we obtain an explicit $u$-independent quantity (which explicitly shows that $u$ is a gauge redundancy)

$$
\begin{aligned}
\operatorname{Tr}\left[V_{c} \psi_{u}\right] & =-\int_{0}^{\infty} d T u\left\langle V_{c}(i \infty) B c \partial c(0) \partial_{T u} \phi_{T u}(0) e^{-\int_{0}^{1} d s \phi_{T u}(s)}\right\rangle_{C_{1}} \\
& =-\int_{0}^{\infty} d y\left\langle V_{c}(i \infty) B c \partial c(0) \partial_{y} \phi_{y}(0) e^{-\int_{0}^{1} d s \phi_{y}(s)}\right\rangle_{C_{1}} .
\end{aligned}
$$

Now we have to get rid of the $B$ line. Remembering that

$$
V_{c}(i \infty)=c(i \infty) c(-i \infty) V_{\text {matter }}(i \infty,-i \infty)
$$

and since $B$ vanishes (fast enough) at the midpoint, we just have ${ }^{10}$

$$
\left\langle V_{c}(i \infty) B c\left(t_{1}\right) c\left(t_{2}\right)\right\rangle_{C_{T}}=\frac{t_{1}}{T}\left\langle V_{c}(i \infty) c\left(t_{2}\right)\right\rangle_{C_{T}}-\frac{t_{2}}{T}\left\langle V_{c}(i \infty) c\left(t_{1}\right)\right\rangle_{C_{T}} .
$$

We thus obtain

$$
\begin{aligned}
\operatorname{Tr}\left[V_{c} \psi_{u}\right] & =+\int_{0}^{\infty} d y 1 \times\left\langle V_{c}(i \infty) c(0) \partial_{y} \phi_{y}(0) e^{-\int_{0}^{1} d s \phi_{y}(s)}\right\rangle_{C_{1}} \\
& =\int_{0}^{\infty} d y\left\langle V_{c}(i \infty) c(0)\left(-\partial_{y}\right) e^{-\int_{0}^{1} d s \phi_{y}(s)}\right\rangle_{C_{1}} \\
& =\int_{0}^{\infty} d y\left(-\partial_{y}\right)\left\langle V_{c}(i \infty) c(0) e^{-\int_{0}^{1} d s \phi_{y}(s)}\right\rangle_{C_{1}} \\
& =\left(\lim _{y \rightarrow 0^{+}}-\lim _{y \rightarrow \infty}\right)\left\langle V_{c}(i \infty) c(0) e^{-\int_{0}^{1} d s \phi_{y}(s)}\right\rangle_{C_{1}} \\
& =\ll V_{c} \gg^{U V}-\ll V_{c} \gg^{I R},
\end{aligned}
$$

as wanted. Notice the importance of (4.5) in order to assure that $\ll V_{c} \gg^{I R} \neq 0$, so that we are not computing a tachyon vacuum observable.

\section{Energy, a general expression for the cubic term}

Assuming the validity of the equation of motion against the solution itself, the energy of a time independent classical solution is given by

$$
E(\psi)=-S[\psi]=\frac{1}{6} \operatorname{Tr}[\psi Q \psi]=-\frac{1}{6} \operatorname{Tr}\left[\psi^{3}\right]
$$

Since two Schwinger parameters are needed to represent $Q \psi$ as a superposition of deformed wedges, we do not get any simplification in computing $\operatorname{Tr}\left[\psi_{u} Q \psi_{u}\right]$ rather then $\operatorname{Tr}\left[\psi_{u}^{3}\right]$. This is because, contrary to the universal tachyon vacuum case, the deformed wedge states are

\footnotetext{
${ }^{10}$ See [10] for a derivation of this, where the role of the closed string insertion is played by the picture changing operator, whose $c$ content is the same as $V_{c}$.
} 
not BRST closed. Under the assumption $\left(c \phi_{u}\right)^{2}=0$, the identity piece in the solution drops from the evaluation of the energy, and we are left with

$$
\begin{aligned}
\operatorname{Tr}\left[\psi_{u}^{3}\right]= & -\operatorname{Tr}\left[\frac{1}{K+\phi_{u}} u \partial_{u} \phi_{u} B c \partial c \frac{1}{K+\phi_{u}} u \partial_{u} \phi_{u} B c \partial c \frac{1}{K+\phi_{u}} u \partial_{u} \phi_{u} B c \partial c\right] \\
= & -\int_{0}^{\infty} d t_{1} d t_{2} d t_{3}\left\langle e^{-\int_{0}^{T} d s \phi_{u}(s)} B c \partial c u \partial_{u} \phi_{u}(T(x+y)) \partial c u \partial_{u} \phi_{u}(T x) \partial c u \partial_{u} \phi_{u}(0)\right\rangle_{C_{T}} \\
= & -\int_{0}^{\infty} d T u^{3} T^{2} \int_{0}^{1} d x \int_{0}^{1-x} d y \\
& \cdot\left\langle e^{-\int_{0}^{T} d s \phi_{u}(s)} B c \partial c \partial_{u} \phi_{u}(T(x+y)) \partial c \partial_{u} \phi_{u}(T x) \partial c \partial_{u} \phi_{u}(0)\right\rangle_{C_{T}},
\end{aligned}
$$

where we have defined (and accordingly changed variables in the integration)

$$
\begin{aligned}
T & \equiv t_{1}+t_{2}+t_{3} \\
x & \equiv \frac{t_{1}}{T} \\
y & \equiv \frac{t_{2}}{T} .
\end{aligned}
$$

Now we rescale $C_{T} \rightarrow C_{1}$ and get

$$
\begin{aligned}
\operatorname{Tr}\left[\psi_{u}^{3}\right]=-\int_{0}^{\infty} & d T u^{3} T^{2} \int_{0}^{1} d x \int_{0}^{1-x} d y \\
\cdot & \left\langle e^{-\int_{0}^{1} d s \phi_{T u}(s)} B c \partial c \partial_{T u} \phi_{T u}(x+y) \partial c \partial_{T u} \phi_{T u}(x) \partial c \partial_{T u} \phi_{T u}(0)\right\rangle_{C_{1}} .
\end{aligned}
$$

As a last step we change variable

$$
q=u T
$$

to get an explicitly $u$-independent expression

$$
\begin{aligned}
\operatorname{Tr}\left[\psi_{u}^{3}\right] & =-\int_{0}^{\infty} d q q^{2} \int_{0}^{1} d x \int_{0}^{1-x} d y F(q, x, y) \\
F(q, x, y) & =\left\langle e^{-\int_{0}^{1} d s \phi_{q}(s)} B c \partial c \partial_{q} \phi_{q}(x+y) \partial c \partial_{q} \phi_{q}(x) \partial c \partial_{q} \phi_{q}(0)\right\rangle_{C_{1}} \\
& =\langle B c \partial c(x+y) \partial c(x) \partial c(0)\rangle_{C_{1}}^{(g h)} \\
& \times\left\langle e^{-\int_{0}^{1} d s \phi_{q}(s)} \partial_{q} \phi_{q}(x+y) \partial_{q} \phi_{q}(x) \partial_{q} \phi_{q}(0)\right\rangle_{C_{1}}^{(m a t t)}
\end{aligned}
$$

In the case of a universal tachyon vacuum solution $\phi_{q}=q$, as it is easy to check, we get back Erler-Schnabl result. But in general, although the structure seems promising, we do not know how to compute the 3-point function in the deformed matter correlator in full generality: for the closed string overlap the matter correlator was just a one-point function in the deformed theory, which could be easily obtained by differentiating the partition function. Here there are three matter+ghost insertions, which upon integration should still give a result related to an appropriate differential operator acting on the partition function. In fact, at the end of the day the correct result should be given by

$$
-\frac{1}{6} \operatorname{Tr}\left[\psi_{u}^{3}\right]=-\frac{1}{2 \pi^{2}}(g(u=0)-g(u=\infty))=-E^{(U V)}+E^{(I R)},
$$


where $g(u)$ is the open string partition function on the canonical cylinder

$$
g(u)=\left\langle e^{-\int_{0}^{1} d s \phi_{u}(s)}\right\rangle_{C_{1}} .
$$

This is because, assuming $\frac{g(u=\infty)}{g(u=0)}=\frac{E^{(I R)}}{E^{(U V)}}$, 14, and knowing that for tachyon vacuum universal solutions we have

$$
E\left(\psi_{T V}\right)=-E^{(D 25)}=-\frac{1}{2 \pi^{2}}\langle 0|| 0\rangle^{m a t t}=-\frac{1}{2 \pi^{2}}\langle 1\rangle=-\frac{1}{2 \pi^{2}}(g(0))^{26}=-\frac{1}{2 \pi^{2}} \prod_{i=0}^{25}\left(\frac{V_{i}}{2 \pi}\right),
$$

this is the only way the correct ratio of tensions can be obtained.

\section{Examples}

In the previous section we constructed a solution based on the seed $\phi_{u}$ subject to the conditions

$$
\begin{aligned}
\left(c \phi_{u}\right)^{2} & =0 \\
f_{t} \circ \phi_{u}(z) & =\frac{1}{t} \phi_{t u}\left(\frac{z}{t}\right) \\
\phi_{u}-\delta \phi_{u} & =u \partial_{u} \phi_{u} .
\end{aligned}
$$

In addition, in order to flow to a well defined IR limit (different from the tachyon vacuum), the partition function

$$
g(u) \equiv\left\langle e^{-\int_{0}^{1} d s \phi_{u}(s)}\right\rangle_{C_{1}}
$$

should obey

$$
\lim _{u \rightarrow \infty} g(u)=Z_{I R}=\text { finite. }
$$

This last constraint, which is also the most important one, is the only one that we cannot satisfy without knowing in advance the exact RG flow on the worldsheet. We now show how some well-known worldsheet RG flows naturally fall inside this scheme.

\subsection{An explicit example: lumps in non-compact dimensions}

A remarkable example (which only lives in the uncompactified limit) is the famous Witten 445] boundary deformation. This deformation was considered by many authors in the context of Boundary String Field Theory, [46], and, more recently, by Ellwood, [39] in a pioneering proposal for Lump Solutions. The deformation (defined in the $\frac{2}{\pi}$ arctan-sliver frame is given by)

$$
\begin{aligned}
\phi_{u}(z) & \equiv u f_{u} \circ\left(: X^{2}:(u z)+A\right)=u\left[: X^{2}:(z)+2(\log u+A)\right], \\
f_{u}(z) & =\frac{z}{u} .
\end{aligned}
$$

The key property of such insertion is that, when exp-integrated on the boundary it interpolates between Neumann $(u=0)$ and Dirichlet $(u=\infty)$, [45]. 
The $u \log u$ piece is there to compensate for the non-primary conformal transformation of : $X^{2}$ :

$$
f \circ: X^{2}(z):=: X^{2}(f(z)):-\log \left|f^{\prime}(z)\right|^{2} .
$$

This implies that under conformal transformations, the parameter $u$ gets changed as follows

$$
f \circ \phi_{u}(z)=\frac{1}{\left|\partial_{z^{\prime}} f^{-1}\left(z^{\prime}\right)\right|} \phi_{u\left|\partial_{z^{\prime}} f^{-1}\left(z^{\prime}\right)\right|}\left(z^{\prime}\right) .
$$

In particular, under scaling $f_{t}(z)=z / t$, we have

$$
f_{t} \circ \phi_{u}(z)=\frac{1}{t} \phi_{t u}\left(\frac{z}{t}\right)
$$

and consequently

$$
f_{t} \circ \int_{a}^{b} d y \phi_{u}(y)=\int_{\frac{a}{t}}^{\frac{b}{t}} d \tilde{y} \phi_{t u}(\tilde{y}) .
$$

The pure number $A$, which depends on the normal ordering conventions (we choose the standard UHP convention), must be determined in order to have a finite partition function in the infrared, see later.

Now we just need to derive $\delta \phi$ by making the BRST variation

$$
\left[Q, \phi_{u}(z)\right]=u\left[Q,: X^{2}(z):\right]=u\left(-2 \partial c(z)+c \partial: X^{2}(z):\right) .
$$

The second piece in the BRST variation does not enter the solution and the first piece (the one proportional to $\partial c$ ) defines $\delta \phi$ to be

$$
\delta \phi_{u}(z)=-2 u
$$

Notice that this is just a number.

In total, one can check that (4.3) is satisfied

$$
\phi_{u}-\delta \phi_{u}=u \partial_{u} \phi_{u}
$$

$A$ is a $u$-independent constant which must be determined in order for the partition function to be finite in the $u \rightarrow \infty$ limit (that is the IR fixed point of the world-sheet boundary deformation). In Appendix D we review this derivation and explicitly find

$$
A=\gamma-1+\log 4 \pi \text {. }
$$

We remark that $\mathrm{A}$ is not just an anonymous integration constant, but represents 'the right amount' of zero momentum tachyon that is necessary to insert in order to get a finite partition function in the IR.

Integrating $\phi_{u}$ on a cylinder $C_{T}$ we get the partition function which is given by, see Appendix D,

$$
\begin{aligned}
\left\langle e^{-\int_{0}^{T} d s \phi_{u}(s)}\right\rangle_{C_{T}} & =\left\langle e^{-\int_{0}^{1} d s \phi_{T u}(s)}\right\rangle_{C_{1}}=g(T u) \\
g(u) & =\frac{1}{\sqrt{2 \pi}} \sqrt{2 u} \Gamma(2 u)\left(\frac{e}{2 u}\right)^{2 u} .
\end{aligned}
$$


In the UV limit $u \rightarrow 0$ we have

$$
\lim _{u \rightarrow 0} g(u)=\lim _{u \rightarrow 0} \frac{1}{2 \sqrt{\pi u}}=\delta(0)=\int_{-\infty}^{\infty} \frac{d x}{2 \pi}=\langle 0|| 0\rangle=\frac{V_{1}}{2 \pi},
$$

while for $u \rightarrow \infty$

$$
\lim _{u \rightarrow \infty} g(u)=\left.\lim _{u \rightarrow \infty} e^{-2 u(\gamma-1+\log 4 \pi-A)}\left(1+O\left(\frac{1}{u}\right)\right)\right|_{A=\gamma-1+\log 4 \pi}=1 .
$$

This indeed reproduces the ratio of tensions (using the fact that the total mass, up to some universal constant, is proportional to the partition function, [4]])

$$
\frac{\tau^{(D 24)}}{\tau^{(D 25)}}=\frac{g(\infty)}{\frac{g(0)}{V_{1}}}=\frac{1}{\frac{1}{2 \pi}}=2 \pi .
$$

Finally we would like to remark that it is not hard to construct $\mathrm{D}(25$-p)-branes, by introducing additional coordinate fields and couplings, starting from $\sum_{i=1}^{p} u_{i}: X_{i}^{2}:(z)$, instead of the simple $u: X^{2}:(z)$ as above. Indeed we know from [45, 46] that the renormalization group flow does not mix up such different couplings, which continue to evolve linearly. As a consequence the construction of D23, D22,..-brane solutions is a straightforward generalization of the one for the D24-brane, see [58] for the explicit construction.

\subsubsection{Multiple lower dimensional branes?}

We saw that having a quadratic minimum in the worldsheet boundary integration places a lower dimensional D-brane at that minimum. A simple generalization consists in taking a $\phi_{u}$ which has several quadratic minima ${ }^{11}$. Then, according to the heuristic property that in the IR the $\mathrm{X}$ field is constrained to stay at the minimum on the boundary potential, we expect to have several displaced D24-branes. Here we just want to point out that, as far as the general structure is concerned, our solution can easily handle such a non-abelian configuration. Concentrating on a case with two distinct quadratic minima we can consider in full generality the relevant field

$$
\chi^{(a)}(z)=:(X-a)^{2}(X+a)^{2}:=: X^{4}:-2 a^{2}: X^{2}:+a^{4}, \quad a>0
$$

The corresponding seed can be taken to be $\left(f_{t}(z)=\frac{z}{t}\right)$

$$
\begin{aligned}
\phi_{u}^{(a)} & =u f_{u} \circ \chi^{(a)}(u z) \\
& =X^{4}(z)+\left(12 \log u-2 a^{2}\right) X^{2}(z)+12 \log ^{2} u-4 a^{2} \log u+a^{4},
\end{aligned}
$$

where use have been made of (normal ordering is understood)

$$
\begin{aligned}
f \circ X^{4}(z) & =\left.\left(-i \partial_{p}\right)^{4} f \circ e^{i p X(z)}\right|_{p=0}=\partial_{p}^{4}\left(\left|f^{\prime}(z)\right|^{p^{2}} e^{i p X(f(z))}\right) \\
& =X^{4}(f(z))-12 \log \left|f^{\prime}(z)\right| X^{2}(f(z))+12 \log ^{2}\left|f^{\prime}(z)\right|
\end{aligned}
$$

\footnotetext{
${ }^{11}$ It appears natural to require the minima to be at the same height although, without explicitly solving the $\mathrm{RG}$ flow near the IR, it is not possible to be more concrete on this point.
} 
Since $f_{t} \circ f_{u}=f_{t u}$, the seed defined in this way will automatically satisfy

$$
f_{t} \circ \phi_{u}(z)=\frac{1}{t} \phi_{t u}\left(\frac{z}{t}\right)
$$

using

$$
\delta X^{n}=\left.\left(-i \partial_{p}\right)^{n} \delta e^{i p X}\right|_{p=0}=\left.\left(-i \partial_{p}\right)^{n} p^{2} e^{i p X}\right|_{p=0},
$$

one can also verify that

$$
\phi_{u}^{(a)}-\delta \phi_{u}^{(a)}=u \partial_{u} \phi_{u}^{(a)}
$$

It is is easy to see that contact term divergences are still logarithmic, which is more than enough to make

$$
\left(c \phi_{u}^{(a)}\right)^{2}=0
$$

and

$$
\operatorname{Tr}\left[e^{-T\left(K+\phi_{u}^{(a)}\right)}(\ldots)\right] \rightarrow\left\langle e^{\left.-\int_{0}^{T} d s \phi_{u}^{(a)}(s)\right)}(\ldots)\right\rangle_{C_{T+(\ldots)}}
$$

without need of regularization/renormalization. This can be of course generalized to coincident branes (just take $a=0$ ), and the number of branes can be straightforwardly increased starting with : $X^{2 n}$ : and 'covariantizing' it by defining

$$
\phi_{u}(z)=u f_{u} \circ: X^{2 n}:(u z)
$$

Unfortunately, it is only for the $X^{2}$ deformation that we can completely solve the worldsheet theory (since in that case it is just a free theory for the $X$ field, with a boundary 'mass' term). In particular, without knowing the partition function in the infrared, we cannot tune the seed $\phi_{u}$ in order to make $\frac{1}{K+\phi}$ singular while $\operatorname{Tr}\left[\frac{1}{K+\phi}(\phi-\delta \phi)\right]$ finite, so this remains as a proposal for further investigations.

\subsection{Other examples: primary seeds}

The simplest example of a seed satisfying the conditions 4.2,4.3 is actually given by taking a primary relevant operator $\chi^{(h)}(z)$ of weight $h<\frac{1}{2}$ (in order to avoid contact term divergences in the solution) and to define

$$
\phi_{u}(z)=u\left(f_{u} \circ \chi^{(h)}(u z)+A(h)\right)=u\left(u^{-h} \chi^{(h)}(z)+A(h)\right) .
$$

Condition (4.2) is satisfied because

$$
f_{t} \circ \chi^{(h)}(z)=t^{-h} \chi^{(h)}(z / t)
$$

Condition (4.3) is satisfied because

$$
(1-\delta) \chi^{(h)}(z)=(1-h) \chi^{(h)}(z)
$$


The pure number $A(h)$ (which will in general depend on the weight $h$ ) should be determined in order for the IR partition function to be finite. This requires to be able to exactly solve the worldsheet theory in the presence of the boundary interaction given by $\int_{\partial M} d s \chi^{(h)}(s)$. This is not known in general except for a few integrable interactions.

The prototype example of this is the celebrated $\cos (X / R)$ deformation (with $R>\sqrt{2}$, because of the technical assumption $(c \phi)^{2}=0$ ), which is known to be exactly solvable, [37, 36]. In this case the solution will describe the flow between a D25 brane wrapped on a circle of radius $\mathrm{R}$ and a D24-brane placed at the minimum of the boundary potential. Let us see in this case how to tune the seed in order to get a finite partition function in the infrared, thus obtaining a solution describing the condensation of a brane wrapping a circle to a lower dimensional brane living at a specific point of the circle.

In [36], first using TBA techniques [37], and then checking in conformal perturbation theory, the following IR limit was derived (which we write down using standard 'String (Field) Theory' convention)

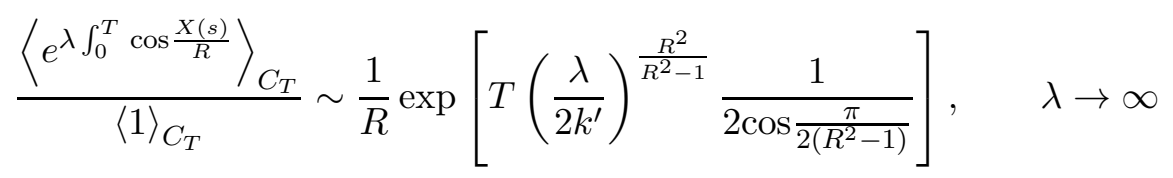

where $k^{\prime}$ is some positive constant (relating the coupling $\lambda$ to the boundary temperature, [36]). Notice that the above expression is well defined for $R^{2}>2$, which is the usual condition which makes the ordered exponential finite without the need of regularization/renormalization.

That's all we need.

In our setting we define the un-tuned seed

$$
\hat{\phi}_{u} \equiv u f_{u} \circ\left(-\cos \frac{X}{R}\right)=-u^{\frac{R^{2}-1}{R^{2}}} \cos \frac{X}{R},
$$

and we are interested in the large $u$ limit of

$$
\frac{\left\langle e^{-\int_{0}^{T} \hat{\phi}_{u}(s)}\right\rangle_{C_{T}}}{\langle 1\rangle_{C_{T}}}
$$

By comparison we have

$$
\lambda=u^{\frac{R^{2}-1}{R^{2}}},
$$

which readily gives

$$
\frac{\left\langle e^{-\int_{0}^{T} \hat{\phi}_{u}(s)}\right\rangle_{C_{T}}}{\langle 1\rangle_{C_{T}}} \sim \frac{1}{R} \exp [\eta(R) T u], \quad u \rightarrow \infty
$$

where we have defined

$$
\eta(R)=\frac{1}{\left(2 k^{\prime}\right)^{2 \frac{R^{2}}{R^{2}-1}} \cos \frac{\pi}{2\left(R^{2}-1\right)}} .
$$


Notice that with our choice of seed the RG-flow is exactly linear ( $u$ increases linearly under scaling), which is a quite non trivial consistency check of the condition (4.2).

Now we tune the seed by adding the appropriate contribution proportional to the identity operator

$$
\phi_{u}=\hat{\phi}_{u}+u A(R)
$$

and we uniquely determine $A(R)$ in order for the partition function to be finite in the IR

$$
A(R)=\eta(R) .
$$

We then have

$$
\lim _{u \rightarrow \infty} \frac{\left\langle e^{-\int_{0}^{T} \phi_{u}(s)}\right\rangle_{C_{T}}}{\langle 1\rangle_{C_{T}}}=\frac{1}{R}=\frac{Z^{I R}}{Z^{U V}} .
$$

This, of course, reproduces the ratio of tensions

$$
\frac{\tau^{D 24}}{\tau^{D 25}}=\operatorname{Vol} \frac{Z^{I R}}{Z^{U V}}=2 \pi R \frac{1}{R}=2 \pi
$$

We would like to point out that the necessity of tuning the seed solves a puzzle raised in 33. about the fact that both cos and - cos give the same (but translated) D24-branes. This, in turn, looked inconsistent with the non-symmetric shape of the OSFT tachyon potential under $T \rightarrow-T$. But from the above analysis it is evident that, after adding the $A(R)$-correction (which, being a number, is usually ignored in the world-sheet analysis as it corresponds to an additive constant in the world-sheet action), the $T \rightarrow-T$ asymmetry is restored and $-\phi_{u}$ will definitely give a divergent partition function in the infrared, telling us that the solution is falling in the unbounded side of the tachyon potential.

\subsubsection{Interpolating from infinite to finite volume}

It is useful to understand the infinite transverse volume as a limit of a circle with increasing radius $R$. Since the : $X^{2}$ : deformation only lives in the strict $R=\infty$ limit, it cannot be used on the circle. In order to define a deformation of it which can live on circles, we observe that

$$
: X^{2}:=-\partial_{p}^{2}: e^{i p X}:\left.\right|_{p=0}
$$

Using

$$
\left.\partial_{p}^{2} f(p)\right|_{p=0}=\lim _{\epsilon \rightarrow 0} \frac{f(\epsilon)-2 f(0)+f(-\epsilon)}{\epsilon^{2}},
$$

and the fact that on a circle of radius $R$ we have $\epsilon=\frac{1}{R}$, because of momentum quantization we get that $X^{2}$ is deformed to

$$
: X^{2}: \quad \rightarrow \quad 2 R^{2}\left(1-: \cos \frac{X}{R}:\right)=: X^{2}:+O\left(\frac{1}{R}\right)
$$


The corresponding seed will thus be given by

$$
\begin{aligned}
\phi_{u}^{(R)} & \equiv u f_{u} \circ\left(2 R^{2}\left(1-: \cos \frac{X}{R}:\right)+A(R)\right) \\
& =-2 u R^{2}\left(u^{-\frac{1}{R^{2}}}: \cos \frac{X}{R}:-1-\frac{A(R)}{R^{2}}\right) .
\end{aligned}
$$

Again, the quantity $A(R)$ should be derived by studying the partition function in the $u \rightarrow \infty$ limit. Using the result of the previous paragraph we get

$$
A(R)=2 R^{2}-\frac{1}{2 \cos \frac{\pi}{2\left(R^{2}-1\right)}}\left(\frac{R^{2}}{k^{\prime}}\right)^{\frac{R^{2}}{R^{2}-1}},
$$

and the ratio of tensions is obviously reproduced. The precise matching with the $A$ determined in the $R=\infty$ limit, (7.15), would require some information about the unknown parameter $k^{\prime}$ and its possible (mild) dependence on $\mathrm{R}$ (in order to cancel the subleading logarithmic divergence of $(7.46)$ in the $R \rightarrow \infty$ limit).

\section{Conclusion and discussion}

The content of our paper can be summarized as follows: given a worldsheet boundary $R G$ flow, generated by the boundary integration of a relevant matter field (whose contact term divergences are mild enough to make $e^{-\int_{a}^{b} d s \phi(s)}$ finite without the need of renormalization), we are able to associate to it a simple solution of OSFT representing the new BCFT which is met at the end of the $R G$ flow.

The solution is defined by the two crucial quantities

$$
\begin{aligned}
& \frac{1}{K+\phi} \\
& \frac{B}{K+\phi}(\phi-\delta \phi) .
\end{aligned}
$$

If the first quantity is regular, then the cohomology of the solution can be trivialized by the well-defined homotopy field

$$
A_{\phi}=\frac{B}{K+\phi}
$$

and the solution will describe the tachyon vacuum. Thus a singular $\frac{1}{K+\phi}$ is needed in order for the solution to describe an IR fixed point where the appropriate open string dynamics is left. This is controlled by the second quantity which, since it enters explicitly in the solution, should be finite in correlators. These two conditions are needed to describe a non trivial IR fixed point.

In principle any $\phi$ such that $\frac{1}{K+\phi}$ is singular while $\frac{B}{K+\phi}(\phi-\delta \phi)$ is finite, can be used to identify a new BCFT, the IR fixed point. However, it is in general not easy to 'tune' $\phi$ so that the two conditions are simultaneously met. 
One of the main result of the paper is the recognition that, for a simple and 'natural' class of seeds (4.2,4.3), these two conditions boil down to the requirement of a finite ( and non vanishing) partition function in the infrared. We indeed find that the trace of $\frac{1}{K+\phi}(\phi-\delta \phi)$ is nothing but the shift in the partition function from the UV to the IR

$$
\operatorname{Tr}\left[\frac{1}{K+\phi}(\phi-\delta \phi)\right]=Z_{\phi}^{(U V)}-Z_{\phi}^{(I R)} .
$$

Thanks to the same mechanism the Ellwood invariants of the solution will correctly compute the corresponding shift in the closed string one-point function.

It would be nice to explicitly compute the boundary state of the solution [47] to verify whether

$$
\left|B_{*}\left(\psi_{u}\right)\right\rangle=?=\lim _{u \rightarrow \infty} e^{-\int_{0}^{2 \pi} \frac{d \theta}{2 \pi} \phi_{u}(\theta)}|B\rangle .
$$

This is expected since the solution is still wedge-based and an explicit check would be instructive, especially in view of the results presented in [55].

For the energy things are not so simple: already for the simplest relevant deformation given by the tachyon vacuum the energy equals the shift in the partition function thanks to a kind of 'miracle'. In fact, after integrating on the first two Schwinger parameters, the energy of the Erler-Schnabl tachyon vacuum turns out to be

$$
\begin{aligned}
E\left(\psi_{T V}\right) & =-\frac{1}{6} \operatorname{Tr}\left[\psi_{T V}^{3}\right]=-\frac{1}{2 \pi^{2}} \int_{0}^{\infty} d t \frac{1}{2} t^{2} e^{-t} \\
& =\frac{1}{2 \pi^{2}} \int_{0}^{\infty} d t \partial_{t} F(t)=\frac{1}{2 \pi^{2}}(F(\infty)-F(0)),
\end{aligned}
$$

where

$$
\begin{aligned}
F(t) & =\frac{1}{2}\left(t^{2}+2 t+2\right) g(t) \\
g(t) & =e^{-t}=\left\langle e^{-t \int_{0}^{2 \pi} \frac{d \theta}{2 \pi}}\right\rangle_{\text {Disk }}=\text { Partition Function. }
\end{aligned}
$$

The energy then equals the shift in the partition function because

$$
\begin{aligned}
F(0) & =g(0)=<1>=\frac{V^{D}}{(2 \pi)^{D}} \\
F(\infty) & =g(\infty)=0
\end{aligned}
$$

In other words the on-shell action is the integral of a total derivative of a function which equals the partition function only 'on shell' (that is at the conformal fixed point). While this is basically what happens in Boundary String Field Theory, we have not set out in the present paper to compute the 'off shell' partition function $F(t)$ for a general relevant deformation. We think however this will be possible by carefully analyzing the involved matter+ghost correlator, (6.5).

Notice that, just because we correctly described the coupling of on-shell closed strings to our solution (and because such coupling is always proportional to the tension), the correct ratio of tensions has been already reproduced. But, needless to say, the consistency 
check that the ratio is the same from the computation of the on-shell action is missing, see [55] and 56].

What is also lacking is a careful analysis of the cohomology around the solution, see [55].

Notwithstanding the many important aspects that still need to be refined and understood in detail, we believe that we provided the first explicit proposal for an analytic solution of OSFT representing a relevant boundary deformation.

We end with a couple of comments. Since very few boundary RG flows are explicitly computable, what we really would like to do is to use OSFT to define the new BCFT which is the result of the (unknown) RG-flow, as it was numerically done in [35]. Unfortunately we do not have very concrete things to say about this. The problem is that, no matter what the relevant operator is that we start with, it is extremely easy to just flow to the tachyon vacuum, or to get a singular solution. The reason for this is essentially the fact that sliver-like projectors (which are the ones that, upon rescaling, give the partition function in the IR) are defined as infinite products of 'wedge states' and, as such, are extremely sensitive to the normalization in front of such wedge states. In our language this means that the relevant operator we use should be supplied with a part proportional to the identity operator, that should be fixed (uniquely) by the requirement of a finite partition function in the infrared. This in turns require to be able to calculate the behavior of the RG flow near the IR. It should be stressed that, ultimately, this problem is linked to the definition of well-defined sliver projectors, rather than the solutions themselves.

It would be certainly desirable to analyze the solution we presented in level expansion, in a convenient conformal frame. However, a genuine $L_{0}$ (such as the one of [35]) or $\mathcal{L}_{0}$ level expansion would be well defined only in a compactified setting in order to have a discrete spectrum for the momentum, which would oblige us to discard the best understood relevant deformation based on $X^{2}$.

One can also wonder if our solution can be obtained by fixing a suitable gauge, and then iteratively solving the equation of motion starting with a relevant field and, correcting it order by order, to end up with a finite solution, as it was done in [48], for the tachyon vacuum. It appears natural that the seed itself will enter the gauge fixing condition, which will be a sort of generalization of the 'dressed' $B$-gauge fixing conditions of [4].

We think these are important aspects to be addressed in the future.

\section{Acknowledgments}

C.M. thanks Ted Erler, Leonardo Rastelli and Martin Schnabl for interesting discussions. We would like to thank the organizers of the workshop APCTP Focus Program on Current Trends in String Field Theory at APCTP, Pohang, South Korea, where this work was started, for the hospitality and for the stimulating environment. C.M. would like to thank SISSA and the Simons Workshop in Mathematics and Physics 2010 for the kind hospitality during part of this research. The work of D.D.T. was supported by the Korean Research Foundation Grant funded by the Korean Government with grant number KRF 
2009-0077423. The research of L.B. was supported in part by the Project of Knowledge Innovation Program (PKIP) of Chinese Academy of Sciences, Grant No. KJCX2.YW.W10.

\section{Appendix}

\section{A. Building the solution from the Tachyon Vacuum}

Here we show that our solution can be alternatively obtained following the construction proposed by Ellwood, [39]. We start with a 'seed' string field $\Gamma$ which is not a solution and use it to construct

$$
\begin{aligned}
\Omega_{L} & =-A \Gamma \\
\Omega_{R} & =-\Gamma A,
\end{aligned}
$$

where $A$ is the homotopy field at the tachyon vacuum

$$
\mathcal{Q} A=1
$$

We can then use $\Omega_{L}$ and $\Omega_{R}$ to build two $\mathrm{L} / \mathrm{R}$ solutions at the tachyon vacuum

$$
\begin{aligned}
\tilde{\psi}_{L} & =\frac{1}{1-\Omega_{L}} \mathcal{Q}\left(1-\Omega_{L}\right)=U_{L}^{-1} \mathcal{Q} U_{L} \\
\tilde{\psi}_{R} & =\left(1-\Omega_{R}\right) \mathcal{Q} \frac{1}{1-\Omega_{R}}=U_{R} \mathcal{Q} U_{R}^{-1} .
\end{aligned}
$$

If $\Gamma$ were a solution, then we would have $\tilde{\psi}_{L}=\tilde{\psi}_{R}=\Gamma$. However $\tilde{\psi}_{L, R}$ are different if $\Gamma$ is not a solution. It is useful to trace them back to the perturbative vacuum, where they will also be expressed in pure gauge form.

$$
\begin{aligned}
& \psi_{L}=\left(U_{L}^{-1} U_{0}\right) Q\left(U_{0}^{-1} U_{L}\right)=V_{L} Q V_{L}^{-1} \\
& \psi_{R}=\left(U_{R} U_{0}\right) Q\left(U_{0}^{-1} U_{R}^{-1}\right)=V_{R}^{-1} Q V_{R} .
\end{aligned}
$$

The tachyon vacuum solution is given by

$$
\psi_{0}=U_{0} Q U_{0}^{-1}
$$

where

$$
U_{0}=1-h F B c F h^{-1}
$$

$h=h(K)$ is a gauge freedom whose only effect is to change the security strips of the tachyon vacuum solution which is ${ }^{12}$

$$
\psi_{0}=h F c \frac{K B}{1-F^{2}} c F h^{-1}
$$

\footnotetext{
${ }^{12}$ To be precise, the gauge freedom given by $h$, changes the tachyon vacuum so, in general, $\tilde{\psi}_{L, R}$ will be solutions around two different, gauge equivalent forms, of the tachyon vacuum. This subtlety is anyhow unimportant when the solutions are brought back to the perturbative vacuum $\psi=0$, as we do.
} 
choosing $h=F^{-1}$ we can put the strip on the right, while for $h=F$ it will be on the left. The homotopy field is independent of $h$ and is given by

$$
A=B f
$$

where we have defined for convenience

$$
f=f(K)=\frac{1-F^{2}(K)}{K} .
$$

This is a very general scheme which is not guaranteed to work in general. Let us now test it for the $D 25$ brane.

\section{A.1 Seeding the D25-brane}

We begin by showing how to get the D25 brane solution, starting from an identity-based seed which is simply

$$
\Gamma=-c
$$

which gives

$$
\begin{aligned}
\Omega_{L} & =f B c \\
\Omega_{R} & =c B f .
\end{aligned}
$$

Following the scheme of the previous section we write directly the solutions at the perturbative vacuum. Concentrating on the left solution

$$
\psi_{L}=\left(U_{L}^{-1} U_{0}\right) Q\left(U_{0}^{-1} U_{L}\right)=V_{L} Q V_{L}^{-1},
$$

we get

$$
V_{L}=1+\frac{f}{1-f} B c-\frac{1}{1-f} h F B c F h^{-1} .
$$

Now we use the gauge freedom of $h$ to write the transformation in canonical form

$$
\begin{aligned}
h & =F \\
V_{L} & =1+\frac{f-F^{2}}{1-f} B c \\
V_{L}^{-1} & =1-\frac{f-F^{2}}{1-F^{2}} B c .
\end{aligned}
$$

In general, we have a non trivial solution (at the perturbative vacuum) when $V_{L}^{-1}$ is singular. In this case it is easy to see that for any "good" $F(K)$ (that is $F(K)=1+\alpha K+$ $O\left(K^{2}\right)$ around $\left.K=0\right)$ both $V_{L}$ and $V_{L}^{-1}$ are well defined. So this solution is genuinely gauge equivalent to the perturbative vacuum. This becomes even more transparent if we write the tachyon vacuum solution in the gauge

$$
F^{2}=f
$$


which uniquely determines

$$
F^{2}(K)=\frac{1}{1+K}
$$

that is the Erler-Schnabl tachyon vacuum solution. In this gauge we have explicitly

$$
\begin{aligned}
& V_{L}=V_{L}^{-1}=1 \\
& \psi_{L}=0 .
\end{aligned}
$$

To find the 'right' solution we proceed in the same way but now we put the external strip of the TV solution to the right. That is we choose $h=F^{-1}$. With straightforward algebra we can write

$$
\begin{aligned}
\psi_{R} & =V_{R}^{-1} Q V_{R} \\
V_{R} & =\frac{1}{1-F^{2}}\left(1+c B \frac{f-F^{2}}{1-f}\right) \rightarrow\left(1+c B \frac{f-F^{2}}{1-f}\right) \\
V_{R}^{-1} & =\left(1-c B \frac{f-F^{2}}{1-F^{2}}\right)\left(1-F^{2}\right) \rightarrow\left(1-c B \frac{f-F^{2}}{1-F^{2}}\right) .
\end{aligned}
$$

The $K$-dependent factors $\left(1-F^{2}\right)^{ \pm 1}$, although singular, are reducible gauge transformations (they do not act) and can thus be dropped. Notice that the right quantities (modulo the reducible gauge transformations) are obtained by reading the left ones form right to left and viceversa. Again it is obvious that both $V_{R}$ and $V_{R}^{-1}$ are well defined, so this is the perturbative vacuum.

\section{A.2 Seeding a lower dimensional brane}

To construct a lower dimensional brane we choose the identity-based seed

$$
\Gamma=-c \tilde{\phi}
$$

where $\tilde{\phi}$ is some relevant matter operator. This gives

$$
\begin{aligned}
& \Omega_{L}=f B c \tilde{\phi} \\
& \Omega_{R}=\tilde{\phi} c B f,
\end{aligned}
$$

In order to get the simplest possible solution we choose Erler-Schnabl Tachyon Vacuum since the beginning.

$$
F^{2}=f=\frac{1}{1+K}
$$

Then the gauge transformations at the perturbative vacuum are given by

$$
\begin{aligned}
V_{L} & =1-\frac{1}{1+K-\tilde{\phi}}(1-\tilde{\phi}) B c \\
V_{L}^{-1} & =1+\frac{1}{K}(1-\tilde{\phi}) B c,
\end{aligned}
$$


which, after the redefinition

$$
\tilde{\phi}=1-\phi
$$

gives the gauge transformation (3.31) of section 3.2. For completeness we also show the solutions $\psi_{L}$ and $\psi_{R}$ in arbitrary $F^{2} \neq f$ gauge,

$$
\begin{gathered}
\psi_{L}=\frac{1}{1-f \tilde{\phi}}\left(F^{2}-f \tilde{\phi}\right)\left(c K B c+c \frac{K}{1-F^{2}}\left(F^{2}-f \tilde{\phi}\right) B c\right) \\
+\frac{1}{1-f \tilde{\phi}}(f[\tilde{\phi}, K] c-f \partial c \delta \tilde{\phi} B c)
\end{gathered}
$$

and

$$
\begin{aligned}
\psi_{R}=(c B K c & \left.+c B\left(F^{2}-\tilde{\phi} f\right) \frac{K}{1-F^{2}} c\right)\left(F^{2}-\tilde{\phi} f\right) \frac{1}{1-\tilde{\phi} f} \\
& +(c[K, \tilde{\phi}] f+c B \partial c \delta \tilde{\phi} f) \frac{1}{1-\tilde{\phi} f}
\end{aligned}
$$

Still $\psi_{R}$ is obtained by reading $\psi_{L}$ from left to right, but when $F^{2} \neq f$ these solutions do not look simple at all. This is another way of appreciating the 'simplicity' of our solution.

\section{B. Explicit real form for the energy}

The energy of a static solution $\psi$ is measured by (is proportional to) the expression $\langle\psi Q \psi\rangle$, or alternatively by $-\langle\psi \psi \psi\rangle$. We find it more convenient to use the latter.

$$
E=-\frac{1}{6}\langle\psi \psi \psi\rangle
$$

To simplify the notation let us set

$$
X=\frac{1}{K+\phi}, \quad H=\phi-\delta \phi
$$

so that

$$
\psi_{L}=c \phi-X H B c K c, \quad \psi_{R}=c \phi-H c K c B X
$$

Of course $B$ commutes with $\phi, X$ and $H$. We have in addition

$$
[B, c K c]=[K, c], \quad\{B,[K, c]\}=0, \quad B c K c B=[K, c] B
$$

and

$$
\{c,[K, c]\}=0, \quad[c, \phi]=0, \quad[c, H]=0, \quad[c K c, H]=0
$$

It is easy to prove that

$$
\begin{aligned}
& \left\langle\psi_{L} \psi_{L} \psi_{L}\right\rangle=-\langle X H B c K c X H B c K c X H B c K c\rangle \\
& =-\langle H B c K c X H B c K c X H B c K c X\rangle=-\langle H c K c X B H c K c X B H c K c X B\rangle \\
& =-\langle H c K c B X H c K c B X H c K c B X\rangle=\left\langle\psi_{R} \psi_{R} \psi_{R}\right\rangle
\end{aligned}
$$


We have used the fact that in those terms where $\phi c$ appears, $c$ is not screened from another $c$ and so, assuming $(c \phi)^{2}=0$, all these terms vanish. Next we have moved $X$ from extreme left to extreme right using the cyclicity of the trace. We have done the same for $B$ and rearranged the terms so as to obtain the third expression above.

Now we will try to write $\left\langle\psi_{L} \psi_{L} \psi_{L}\right\rangle=\left\langle\psi_{R} \psi_{R} \psi_{R}\right\rangle$ in L-R symmetric form. To this end we write

$$
B c K c=\frac{1}{2}(B c K c+c K c B)+\frac{1}{2}[K, c] .
$$

Then

$$
\begin{aligned}
& \left\langle\psi_{R} \psi_{R} \psi_{R}\right\rangle= \\
= & -\frac{1}{8}\langle\sqrt{X}(B c K c+c K c B) H \sqrt{X} \sqrt{X}(B c K c+c K c B) H \sqrt{X} \sqrt{X}(B c K c+c K c B) H \sqrt{X}\rangle \\
= & -\frac{1}{8}\langle[K, c] H X[K, c] H X[K, c] H X\rangle \\
= & -\frac{3}{8}\langle B c K c H X[K, c] H X[K, c] H X\rangle \\
= & -\frac{3}{8}\langle c K c B H X[K, c] H X[K, c] H X\rangle \\
= & -\frac{3}{8}\langle B c K c H X B c K c H X[K, c] H X\rangle \\
= & -\frac{3}{8}\langle c K c B H X c K c B H X[K, c] H X\rangle
\end{aligned}
$$

In the last two lines we have used the collision of two $B$ 's (and the cyclicity property) to get rid of two additional terms.

The RHS expression of the first line is already in L-R symmetric form, so we leave it there. Now consider $\langle[K, c] H X[K, c] H X c K c B H X\rangle$. We can move $B$ around using cyclicity and (B.4) and get

$$
\begin{aligned}
& \langle[K, c] H X[K, c] H X c K c B H X\rangle=\langle[K, c] H X[K, c] H X B c K c H X\rangle= \\
& \langle[K, c] H X[K, c] H X(c K c B+[K, c]) H X\rangle
\end{aligned}
$$

This means in particular that

$$
\langle[K, c] H X[K, c] H X[K, c] H X\rangle=0
$$

So the second line in the RHS of (B.8) vanishes. Let us consider next the fourth and last line of (B.8)

$$
\begin{aligned}
& \langle c K c B H X[K, c] H X[K, c] H X\rangle+\langle c K c B H X c K c B H X[K, c] H X\rangle \\
& \langle c K c B H X[K, c] H X[K, c] H X\rangle+\langle c K c H X B c K c B H X[K, c] H X\rangle \\
& \langle c K c B H X[K, c] H X[K, c] H X\rangle+\langle c K c H X[K, c] B H X[K, c] H X\rangle \\
& \langle c K c B H X[K, c] H X[K, c] H X\rangle-\langle c K c B H X[K, c] H X[K, c] H X\rangle=0 .
\end{aligned}
$$


What remains to be considered is

$$
\begin{aligned}
& \langle B c K c H X[K, c] H X[K, c] H X\rangle+\langle B c K c H X B c K c H X[K, c] H X\rangle \\
& =\langle B c K c H X[K, c] H X[K, c] H X\rangle-\langle c K c H X B c K c B H X[K, c] H X\rangle \\
& =\langle B c K c H X[K, c] H X[K, c] H X\rangle-\langle c K c H X[K, c] B H X[K, c] H X\rangle \\
& =\langle B c K c H X[K, c] H X[K, c] H X\rangle+\langle c K c B H X[K, c] H X[K, c] H X\rangle \\
& =\langle\{B, c K c\} H X[K, c] H X[K, c] H X\rangle \\
& =-\langle[K, c] H X\{B, c K c\} H X[K, c] H X\rangle .
\end{aligned}
$$

The last expression can be written as

$-\langle[K, c] H X\{B c K c, c K c B\} H X[K, c] H X\rangle=-\langle\sqrt{X}[K, c] H X\{B, c K c\} H X[K, c] H \sqrt{X}\rangle$ and it is L-R symmetric.

Finally

$$
\begin{aligned}
& -\left\langle\psi_{R} \psi_{R} \psi_{R}\right\rangle= \\
= & \frac{1}{8}\langle\sqrt{X}(B c K c+c K c B) H \sqrt{X} \sqrt{X}(B c K c+c K c B) H \sqrt{X} \sqrt{X}(B c K c+c K c B) H \sqrt{X}\rangle \\
& +\langle\sqrt{X}[K, c] H \sqrt{X} \sqrt{X}\{B, c K c\} H \sqrt{X} \sqrt{X}[K, c] H \sqrt{X}\rangle
\end{aligned}
$$

which is LR symmetric, and therefore real.

\section{Cubic Superstring generalization}

As it was the case for the Tachyon Vacuum, [10], our lump solution can also be easily generalized to a corresponding solution in the cubic SSFT. The solution has the same pure gauge form as in the bosonic case, but now $Q$ acts differently. In particular

$$
Q c=c K c-\gamma^{2} .
$$

The BRST variation of the seed $\phi(X)$ is given by

$$
\begin{gathered}
{[Q, \phi(w)]=c \partial \phi(w)+\partial c \delta \phi(w)+\gamma\left(\psi \delta^{\prime} \phi\right)(w),} \\
Q \phi=c[K, \phi]+[K, c] \delta \phi(w)+\gamma\left(\psi \delta^{\prime} \phi\right) .
\end{gathered}
$$

Assuming that the seed $\phi$ is solely made of the $X$ field we have

$$
\begin{aligned}
\delta \phi(X) & =-\partial_{X}^{2} \phi(X) \\
\delta^{\prime} \phi(X) & =-\partial_{X} \phi(X) .
\end{aligned}
$$

The singular gauge transformation which generates the solution is the same as the bosonic one

$$
\begin{aligned}
V_{L} & =1-\frac{1}{K+\phi} \phi B c \\
V_{L}^{-1} & =1+\frac{1}{K} \phi B c .
\end{aligned}
$$


Due to the different action of the BRST charge the solution will contain superstring corrections

$$
\begin{aligned}
\psi_{L}=V_{L} Q V_{L}^{-1} & =c \phi-\frac{1}{K+\phi} B Q(c \phi) \\
& =c \phi-\frac{1}{K+\phi}\left[(\phi-\delta \phi) B c \partial c+\left(\psi \cdot \delta^{\prime} \phi\right) B c \gamma-B \gamma^{2} \phi\right] .
\end{aligned}
$$

Again, the cohomology of the solution can be formally trivialized (the superstring corrections add up to zero)

$$
\mathcal{Q}_{\psi_{L}} \frac{B}{K+\phi}=1
$$

so a singular $\frac{1}{K+\phi}$ is needed to have a solution in a different gauge orbit from the superstring 'tachyon vacuum' of [10].

In this case, however, the regularity of the solution involves different 'sectors' labeled by their ghost structure. It would be interesting to understand if one can find explicit examples of seeds giving rise to solutions different from the tachyon vacuum. Notice that the solution is in the $\mathrm{GSO}(+)$ sector so, if it can be made regular, it would probably describe a codimension 1 'brane' in the $\mathrm{GSO}(+)$ sector, which does not seem to exist in string theory. On the other hand, the impossibility of building a regular solution (with a seed in the $\operatorname{GSO}(+)$ sector) would be consistent with the spectrum of string theory. We think this is an interesting problem which can contribute to the long debate about the consistency of the cubic RNS theory [49, 50, 51, 52, 53, 54].

\section{Computations for the $: X^{2}$ : deformation}

In order to use Witten's results, [45], we have to map the cylinder to the unit disk. Given a cylinder of width $T$ we first scale it to a canonical cylinder of width 1 and obtain

$$
\left\langle e^{-\int_{0}^{T} d s\left[u\left(X^{2}(s)+2 \log u+2 A\right)\right]}\right\rangle_{C_{T}}=\left\langle e^{-\int_{0}^{1} d s\left[T u\left(X^{2}(s)+2 \log T u+2 A\right)\right]}\right\rangle_{C_{1}} .
$$

Now we can map to the unit disk with

$$
w=-e^{2 \pi i \tilde{z}}
$$

where $w$ is the global coordinate on the unit disk and $\tilde{z}$ the global coordinate on the canonical cylinder of width 1 . Explicitly we get

$$
g_{A}(u) \equiv\left\langle e^{-\int_{0}^{1} d s\left[u\left(X^{2}(s)+2 \log u+2 A\right)\right]}\right\rangle_{C_{1}}=\left\langle e^{-\int_{0}^{2 \pi} d \theta\left[\frac{u}{2 \pi}\left(X^{2}\left(e^{i \theta}\right)+2 \log \frac{u}{2 \pi}+2 A\right)\right]}\right\rangle_{D i s k} .
$$

Now to explicitly evaluate (D.3) we use the result of [15], keeping in mind that in there $\alpha^{\prime}=2$ while here we use the more common $\alpha^{\prime}=1$. Thus we have

$$
Z(u) \equiv\left\langle e^{-\int_{0}^{2 \pi} d \theta \frac{u}{4 \pi} X^{2}\left(e^{i \theta}\right)}\right\rangle_{D i s k}=K \sqrt{u} \exp (\gamma u) \Gamma(u)
$$


Here $K$ is a $u$-independent normalization constant, which depends on the way one normalizes the zero mode $x$ integration. We normalize it as follows:

$$
\int_{0}^{\infty} \frac{d x}{2 \pi}=\langle 0|| 0\rangle=\frac{V_{1}}{2 \pi}
$$

So, in our conventions, we have

$$
K=\frac{1}{\sqrt{2 \pi}}
$$

Therefore, the partition function on canonical cylinder in (D.3) is

$$
\left\langle e^{-\int_{0}^{1} \phi_{u}(s) d s}\right\rangle_{C_{1}}=\frac{1}{\sqrt{2 \pi}} \sqrt{2 u} \Gamma(2 u) e^{u\left(2 \gamma-2 \log \frac{u}{2 \pi}-2 A\right)} .
$$

Now we have to determine the $u$-independent number $A$ in order for the partition function to be finite in the $u \rightarrow \infty$ limit. Using Stirling approximation we have

$$
\sqrt{u} \Gamma(u) e^{-u \log u} \approx_{u \rightarrow \infty} \sqrt{2 \pi} e^{-u},
$$

which implies

$$
\lim _{u \rightarrow \infty}\left\langle e^{-\int_{0}^{1} \phi_{u}(s) d s}\right\rangle_{C_{1}}=e^{2 u(\gamma-1+\log 4 \pi-A)} .
$$

The only way this can be finite in the large $u$ limit is

$$
A=\gamma-1+\log 4 \pi
$$

Any other choice of $A$ would give a divergent or vanishing partition function in the infrared. This result is a little bit different from [39] by the $\log 4 \pi$ term, because in [39] of some different conventions.

With this unique choice of $A$ we get

$$
\begin{aligned}
g(u) \equiv & \left\langle e^{-\int_{0}^{1} \phi_{u}(s) d s}\right\rangle_{C_{1}}=\frac{1}{\sqrt{2 \pi}} \sqrt{2 u} \Gamma(2 u)\left(\frac{e}{2 u}\right)^{2 u} \\
& \left\langle e^{-\int_{0}^{T} \phi_{u}(s) d s}\right\rangle_{C_{T}}=g(T u) .
\end{aligned}
$$

In the deep IR we get a properly normalized partition function for Dirichlet boundary conditions

$$
\lim _{u \rightarrow \infty} g(u)=K \sqrt{2 \pi}=\frac{1}{\sqrt{2 \pi}} \sqrt{2 \pi}=1,
$$

while in the UV (no deformation present) we get the partition function with Neumann boundary condition, which is the BCFT on which the theory is defined from the start

$$
\lim _{u \rightarrow 0} g(u)=\lim _{u \rightarrow 0} \frac{1}{2 \sqrt{\pi u}}=\delta(0)=\int_{0}^{\infty} \frac{d x}{2 \pi}=\frac{V_{1}}{2 \pi}=\langle 0|| 0\rangle_{S L(2, R)} .
$$

This last divergence is expected because the $X^{2}$ deformation cannot be defined on a compact direction and all computations are done at infinite transverse volume. The vacuum to vacuum amplitude is indeed divergent $(\delta(p=0))$ because of the non compact zero mode of $X$. 


\section{D.1 Explicit results for one-point function}

In addition to (D.4), from 45, we can also find the following one-point function

$$
\left\langle X^{2} e^{-\int_{0}^{2 \pi} d \theta \frac{u}{4 \pi} X^{2}(\theta)}\right\rangle_{D i s k}=-2 \partial_{u} Z(u)=\left(\frac{1}{u}-2 H(u)\right) Z(u) .
$$

where $H(z) \equiv H_{z}$ is the $z^{\text {th }}$ Harmonic Number. They can be defined as

$$
H(z) \equiv H_{z} \equiv \gamma+\psi(z+1)
$$

It inherits from the digamma $(\psi(z))$ function the following property

$$
H(z)=\frac{1}{z}+H(z-1)
$$

We can then properly map this one point function to the cylinder $C_{T}$ so as to evaluate the one point function of the insertion $\phi_{u}(z)=u\left(X^{2}(z)+2 \log u+2 A\right)$ on the cylinder. It is given by

$$
\left\langle\phi_{u} e^{-\int_{0}^{T} d s \phi_{u}(s)}\right\rangle_{C_{T}}=2 u g_{A}(T u)\left(\frac{1}{4 T u}-H(2 T u)+\log \frac{T u}{2 \pi}+A\right)
$$

where $A$ is determined as in the previous subsection. A non trivial consistency check of the chain of mappings and conventions is given by

$$
\begin{aligned}
\left\langle u \partial_{u} \phi_{u} e^{-\int_{0}^{T} d s \phi_{u}(s)}\right\rangle_{C_{T}} & =\left\langle\left(\phi_{u}+2 u\right) e^{-\int_{0}^{T} d s \phi_{u}(s)}\right\rangle_{C_{T}} \\
& =2 u g_{A}(T u)\left(\frac{1}{4 T u}-H(2 T u)+\log \frac{T u}{2 \pi}+A+1\right)=-\frac{u}{T} \partial_{u} g_{A}(T u),
\end{aligned}
$$

where we have used the identity

$$
\frac{-\frac{u}{T} \partial_{u} g_{A}(T u)}{2 u g_{A}(T u)}=\left(\frac{1}{4 T u}-H(2 T u)+\log \frac{T u}{2 \pi}+A+1\right)
$$

which can easily be verified by the use of the properties of Harmonic Numbers or Digamma functions. We realize that (D.18) is the expression of the boundary changing mechanism at work in section 4

$$
\begin{aligned}
\left\langle\left(\phi_{u}+2 u\right) e^{-\int_{0}^{T} d s \phi_{u}(s)}\right\rangle_{C_{T}} & =\left\langle\left(u \partial_{u} \phi_{u}\left(s^{\prime}\right)\right) e^{-\int_{0}^{T} d s \phi_{u}(s)}\right\rangle_{C_{T}} \\
& =-\frac{u}{T} \partial_{u}\left\langle e^{-\int_{0}^{T} d s \phi_{u}(s)}\right\rangle_{C_{T}}
\end{aligned}
$$

\section{D.2 Three-point functions: energy}

In section 6 we have obtained a general expression of the cubic term in the action which involves a three-point function of $\partial_{u} \phi_{u}$. In this subsection we evaluate it for the solution generated by the insertion $\phi_{u}(z)=u\left(X^{2}(z)+2 \log u+2 A\right)$. We will make use of the 
Green's function in the presence of the boundary deformation $\int_{0}^{2 \pi} d \theta \frac{u}{4 \pi \alpha^{\prime}} X^{2}(\theta)$, which was derived in [45]. On the disc it is given by

$$
\begin{aligned}
G_{u}(z-w) & =\langle X(z, \bar{z}) X(w, \bar{w})\rangle_{\text {Disk }}^{(u)} \\
& =-\frac{\alpha^{\prime}}{2}\left(\ln |z-w|^{2}+\ln |1-z \bar{w}|^{2}-\frac{2}{u}+2 u \sum_{k=1}^{\infty} \frac{(z \bar{w})^{k}+(\bar{z} w)^{k}}{k(k+u)}\right),
\end{aligned}
$$

where we denoted the deformed correlator

$$
\langle(\ldots)\rangle_{D i s k}^{(u)} \equiv\left\langle(\ldots) e^{\int_{0}^{2 \pi} d \theta \frac{u}{4 \pi \alpha^{\prime}} X^{2}(\theta)}\right\rangle_{D i s k}
$$

On the boundary of the disc $\left(z=e^{i \theta}\right)$ we have

$$
\begin{aligned}
G_{u}\left(\theta-\theta^{\prime}\right) & =\sum_{k=-\infty}^{\infty} \frac{e^{i k\left(\theta-\theta^{\prime}\right)}}{|k|+u}=\frac{1}{u}+2 \sum_{k=1}^{\infty} \frac{1}{k+u} \cos k\left(\theta-\theta^{\prime}\right), \\
& =\frac{1}{u}+\frac{2}{1+u} \operatorname{Re}\left[e^{i\left(\theta-\theta^{\prime}\right)}{ }_{2} F_{1}\left(1+u, 1,2+u ; e^{i\left(\theta-\theta^{\prime}\right)}\right)\right]
\end{aligned}
$$

where we have used $\alpha^{\prime}=1$, and ${ }_{2} F_{1}$ is the hypergeometric function

$$
\begin{aligned}
{ }_{2} F_{1}(a, b, c ; z) & ={ }_{2} F_{1}(b, a, c ; z)=\sum_{k=0}^{\infty} \frac{(a)_{k}(b)}{(c)_{k}} \frac{z^{k}}{k !} \\
(x)_{k} & \equiv \frac{\Gamma(x+k)}{\Gamma(x)} .
\end{aligned}
$$

Using this Green's function and the Wick's theorem, in addition to the 1-point function given in the previous section, we obtain the following two- and three-point functions for $X^{2}(\theta)$

$$
\begin{aligned}
& \left\langle X^{2}(\theta)\right\rangle_{D i s k}^{(u)}=Z(u)\left(\frac{1}{u}-2 H(u)\right) \equiv Z(u) h_{u}, \\
& \left\langle X^{2}(\theta) X^{2}\left(\theta^{\prime}\right)\right\rangle_{D i s k}^{(u)}=Z(u)\left(2 G_{u}^{2}\left(\theta-\theta^{\prime}\right)+h_{u}^{2}\right) \\
& \left\langle X^{2}\left(\theta_{1}\right) X^{2}\left(\theta_{2}\right) X^{2}\left(\theta_{3}\right)\right\rangle_{D i s k}^{(u)}=Z(u)\left\{8 G_{u}\left(\theta_{1}-\theta_{2}\right) G_{u}\left(\theta_{1}-\theta_{3}\right) G_{u}\left(\theta_{2}-\theta_{3}\right)\right. \\
& \left.\quad+2 h_{u}\left(G_{u}^{2}\left(\theta_{1}-\theta_{2}\right)+G_{u}^{2}\left(\theta_{1}-\theta_{3}\right)+G_{u}^{2}\left(\theta_{2}-\theta_{3}\right)\right)+h_{u}^{3}\right\}
\end{aligned}
$$

The cubic term is proportional to

$$
\begin{aligned}
& \left\langle\psi_{u} \psi_{u} \psi_{u}\right\rangle=-\left\langle\frac{1}{K+\phi_{u}}\left(\phi_{u}+2 u\right) B c K c \frac{1}{K+\phi_{u}}\left(\phi_{u}+2 u\right) B c K c \frac{1}{K+\phi_{u}}\left(\phi_{u}+2 u\right) B c K c\right\rangle \\
& =-\int_{0}^{\infty} d t_{1} d t_{2} d t_{3} \mathcal{E}_{0}\left(t_{1}, t_{2}, t_{3}\right)\left\langle\left(\phi_{u}\left(t_{1}+t_{2}\right)+2 u\right)\left(\phi_{u}\left(t_{1}\right)+2 u\right)\left(\phi_{u}(0)+2 u\right) e^{-\int_{0}^{T} d s \phi_{u}(s)}\right\rangle_{C_{T}}
\end{aligned}
$$

where $\mathcal{E}_{0}\left(t_{1}, t_{2}, t_{3}\right)$ is the contribution of the ghost sector and $T=t_{1}+t_{2}+t_{3}$. To use (D.24) we have to map the $C_{T}$ to the unit disc by

$$
w(z)=-e^{\frac{2 \pi i z}{T}} .
$$


Then we get,

$$
\begin{aligned}
\left\langle\psi_{u} \psi_{u} \psi_{u}\right\rangle & =-\int_{0}^{\infty} d t_{1} d t_{2} d t_{3} \mathcal{E}_{0}\left(t_{1}, t_{2}, t_{3}\right) e^{-2 u T\left(\ln \left(\frac{u T}{2 \pi}\right)+A\right)} \\
& \times u^{3}\left\langle\left(X^{2}\left(\theta_{t_{1}+t_{2}}\right)+2\left(\ln \left(\frac{u T}{2 \pi}\right)+A+1\right)\right)\left(X^{2}\left(\theta_{t_{1}}\right)+2\left(\ln \left(\frac{u T}{2 \pi}\right)+A+1\right)\right)\right. \\
& \left.\times\left(X^{2}(0)+2\left(\ln \left(\frac{u T}{2 \pi}\right)+A+1\right)\right) e^{-\int_{0}^{2 \pi} d \theta \frac{2 u T}{4 \pi} X^{2}(\theta)}\right\rangle_{D i s k},
\end{aligned}
$$

where $\theta_{t}=\frac{2 \pi t}{T}$. Using (D.24), setting $A=\gamma-1+\ln 4 \pi$ and simplifying,

$$
\begin{aligned}
\left\langle\psi_{u} \psi_{u} \psi_{u}\right\rangle= & -\int_{0}^{\infty} d t_{1} d t_{2} d t_{3} \mathcal{E}_{0}\left(t_{1}, t_{2}, t_{3}\right) u^{3} e^{-2 u T(\ln (2 u T)+\gamma-1)} Z(2 u T) \\
& \cdot\left\{8\left(\frac{h_{2 u T}}{2}+\ln (2 u T)+\gamma\right)^{3}\right. \\
+ & 4\left(\frac{h_{2 u T}}{2}+\ln (2 u T)+\gamma\right)\left(G_{2 u T}^{2}\left(\frac{2 \pi t_{1}}{T}\right)+G_{2 u T}^{2}\left(\frac{2 \pi\left(t_{1}+t_{2}\right)}{T}\right)+G_{2 u T}^{2}\left(\frac{2 \pi t_{2}}{T}\right)\right) \\
+ & \left.8 G_{2 u T}\left(\frac{2 \pi t_{1}}{T}\right) G_{2 u T}\left(\frac{2 \pi\left(t_{1}+t_{2}\right)}{T}\right) G_{2 u T}\left(\frac{2 \pi t_{2}}{T}\right)\right\} .
\end{aligned}
$$

Applying the identity (D.19) we get

$$
\begin{aligned}
\left\langle\psi_{u} \psi_{u} \psi_{u}\right\rangle & =-\int_{0}^{\infty} d t_{1} d t_{2} d t_{3} \mathcal{E}_{0}\left(t_{1}, t_{2}, t_{3}\right) u^{3} g(u T)\left\{8\left(-\frac{1}{2} \frac{\partial_{u T} g(u T)}{g(u T)}\right)^{3}\right. \\
& +4\left(-\frac{1}{2} \frac{\partial_{u T} g(u T)}{g(u T)}\right)\left(G_{2 u T}^{2}\left(\frac{2 \pi t_{1}}{T}\right)+G_{2 u T}^{2}\left(\frac{2 \pi\left(t_{1}+t_{2}\right)}{T}\right)+G_{2 u T}^{2}\left(\frac{2 \pi t_{2}}{T}\right)\right) \\
& \left.+8 G_{2 u T}\left(\frac{2 \pi t_{1}}{T}\right) G_{2 u T}\left(\frac{2 \pi\left(t_{1}+t_{2}\right)}{T}\right) G_{2 u T}\left(\frac{2 \pi t_{2}}{T}\right)\right\}
\end{aligned}
$$

In section 6 we have shown that the above quantity is $u$ independent. We can easily confirm this by doing a convenient change of variables $\left(t_{1}, t_{2}, t_{3}\right) \rightarrow(T, x, y)$, where

$$
\begin{aligned}
& x=\frac{t_{1}}{T} \\
& y=\frac{t_{2}}{T} .
\end{aligned}
$$

The matter part, (D.28), (before integration) can be written as

$$
u^{3} F(u T, x, y),
$$

where

$$
\begin{aligned}
F(u T, x, y) & =g(u T)\left\{8\left(-\frac{1}{2} \frac{\partial_{u T} g(u T)}{g(u T)}\right)^{3}+8 G_{2 u T}(2 \pi x) G_{2 u T}(2 \pi(x+y)) G_{2 u T}(2 \pi y)\right. \\
& \left.+4\left(-\frac{1}{2} \frac{\partial_{u T} g(u T)}{g(u T)}\right)\left(G_{2 u T}^{2}(2 \pi x)+G_{2 u T}^{2}(2 \pi(x+y))+G_{2 u T}^{2}(2 \pi y)\right)\right\}(\mathrm{D} .3
\end{aligned}
$$


Computing the ghost correlator we have

$$
\mathcal{E}_{0}\left(t_{1}, t_{2}, t_{3}\right)=\left\langle B c \partial c\left(t_{1}+t_{2}\right) \partial c\left(t_{1}\right) \partial c(0)\right\rangle_{C_{T}}=\mathcal{E}(x, y)=-\frac{4}{\pi} \sin \pi x \sin \pi y \sin \pi(x+y),
$$

notice that the ghost correlator only depends on $x$ and $y$, which are scale invariant coordinates.

We now change variables of integrations,

$$
\int_{0}^{\infty} d t_{1} \int_{0}^{\infty} d t_{2} \int_{0}^{\infty} d t_{3}=\int_{0}^{\infty} d T T^{2} \int_{0}^{1} d x \int_{0}^{1-x} d y
$$

so, collecting everything, we obtain

$$
-\frac{1}{6} \operatorname{Tr}\left[\psi_{u} \psi_{u} \psi_{u}\right]=\frac{1}{6} \int_{0}^{\infty} d T T^{2} \int_{0}^{1} d x \int_{0}^{1-x} d y \mathcal{E}(x, y) u^{3} F(u T, x, y) .
$$

As a last step we do the obvious change of coordinate

$$
t=u T
$$

which readily gives

$$
-\frac{1}{6} \operatorname{Tr}\left[\psi_{u} \psi_{u} \psi_{u}\right]=\frac{1}{6} \int_{0}^{\infty} d t t^{2} \int_{0}^{1} d x \int_{0}^{1-x} d y \mathcal{E}(x, y) F(t, x, y) .
$$

Notice that $u$ has completely disappeared, so the energy is $u$-independent. In the UV region $t \rightarrow 0$, the integrand goes like $1 / t^{3 / 2} \sim \partial_{t} 1 / \sqrt{t}$, which is the expected volume divergence. As far as the IR $(t \rightarrow \infty)$ is concerned, the finiteness of (D.35) has been proven in [56].

\section{E. Ordered exponential insertion}

In this appendix we show how to write down the insertion $\frac{1}{K+\phi}$ inside a correlator by a Schwinger-like representation. Let us start from the parametric representation

$$
\frac{1}{K+\phi}=\int_{0}^{\infty} d t e^{-t(K+\phi)}
$$

To make sense of this formula we need to split the exponential. The RHS can be understood as the action of the operator $K^{\prime} \sim K_{1}^{L}$ and the field operator $\phi^{\prime}$ on the identity string field,

$$
\int_{0}^{\infty} d t e^{-t(K+\phi)}=\int_{0}^{\infty} d t e^{-t\left(K^{\prime}+\phi^{\prime}\right)}|I\rangle
$$

We will split the operator $e^{-t\left(K^{\prime}+\phi^{\prime}\right)}$ in two exponentials. To this end we use the Zassenhaus formula, which holds for finite matrices,

$$
e^{t(X+Y)}=e^{t X} e^{t Y} e^{-\frac{t^{2}}{2}[X, Y]} e^{\frac{t^{3}}{3 !}(2[Y,[X, Y]]+[X,[X, Y]])} e^{-\frac{t^{4}}{4 !}([[[X, Y], X], X]+3[[[X, Y], X], Y]+3[[[X, Y], Y], Y])} \ldots,
$$

and extend it to general operators. 
Choosing $X=-K^{\prime}, Y=-\phi^{\prime}$, inside a correlator we can assume $[[X, Y], Y]=0$, $[[[X, Y], X], Y]=0$, etc. Therefore the formula simplifies to

$$
e^{-t\left(K^{\prime}+\phi^{\prime}\right)}=e^{-t K^{\prime}} e^{-S(t)}
$$

where

$$
S(t)=t \phi^{\prime}+\frac{t^{2}}{2}\left[K^{\prime}, \phi^{\prime}\right]+\frac{t^{3}}{3 !}\left[K^{\prime},\left[K^{\prime}, \phi^{\prime}\right]\right]+\frac{t^{4}}{4 !}\left[K^{\prime},\left[K^{\prime},\left[K^{\prime}, \phi^{\prime}\right]\right]\right]+\ldots,
$$

with $S(0)=0$. Now, $\phi^{\prime}$ denotes the field $\phi^{\prime}$ evaluated at some point $t_{0}$. Thus

$$
\begin{aligned}
\partial_{t} S(t) & =\phi^{\prime}\left(t_{0}\right)+t\left[K^{\prime}, \phi^{\prime}\left(t_{0}\right)\right]+\frac{t^{2}}{2 !}\left[K^{\prime},\left[K^{\prime}, \phi^{\prime}\left(t_{0}\right)\right]\right]+\frac{t^{3}}{3 !}\left[K^{\prime},\left[K^{\prime},\left[K^{\prime}, \phi^{\prime}\left(t_{0}\right)\right]\right]\right]+\ldots \\
& =\phi^{\prime}\left(t+t_{0}\right)
\end{aligned}
$$

and

$$
S(t)=\int_{t_{0}}^{t+t_{0}} d s \phi^{\prime}(s)
$$

Applying back (E.3) to the identity string field, we obtain

$$
\frac{1}{K+\phi}=\int_{0}^{\infty} d t e^{-t K} e^{-\int_{t_{0}}^{t+t_{0}} d s \phi(s)}
$$

\section{References}

[1] E. Witten, Noncommutative Geometry And String Field Theory, Nucl. Phys. B 268 (1986) 253.

[2] M. Schnabl, Analytic solution for tachyon condensation in open string field theory, Adv. Theor. Math. Phys. 10 (2006) 433 [arXiv:hep-th/0511286].

[3] Y. Okawa, Comments on Schnabl's analytic solution for tachyon condensation in Witten's open string field theory, JHEP 0604 (2006) 055 [arXiv:hep-th/0603159].

[4] T. Erler and M. Schnabl, A Simple Analytic Solution for Tachyon Condensation, arXiv:0906.0979 [hep-th].

[5] L. Rastelli and B. Zwiebach, Solving open string field theory with special projectors, arXiv:hep-th/0606131.

[6] Y. Okawa, L. Rastelli and B. Zwiebach, Analytic solutions for tachyon condensation with general projectors, arXiv:hep-th/0611110.

[7] E. Fuchs and M. Kroyter, On the validity of the solution of string field theory, JHEP 0605 (2006) 006 [arXiv:hep-th/0603195].

[8] T. Erler, Split string formalism and the closed string vacuum, JHEP 0705, 083 (2007) [arXiv:hep-th/0611200].

[9] T. Erler, Split string formalism and the closed string vacuum. II, JHEP 0705, 084 (2007) [arXiv:hep-th/0612050]. 
[10] T. Erler, Tachyon Vacuum in Cubic Superstring Field Theory, JHEP 0801 (2008) 013 [arXiv:0707.4591 [hep-th]].

[11] E. A. Arroyo, Generating Erler-Schnabl-type Solution for Tachyon Vacuum in Cubic Superstring Field Theory, arXiv:1004.3030 [hep-th].

[12] S. Zeze, Tachyon potential in KBc subalgebra, arXiv:1004.4351 [hep-th].

[13] S. Zeze, Regularization of identity based solution in string field theory, arXiv:1008.1104 [hep-th].

[14] E. A. Arroyo, Comments on regularization of identity based solutions in string field theory, arXiv:1009.0198 [hep-th].

[15] M. Kiermaier, Y. Okawa, L. Rastelli and B. Zwiebach, Analytic solutions for marginal deformations in open string field theory, arXiv:hep-th/0701249.

[16] M. Schnabl, Comments on marginal deformations in open string field theory, arXiv:hep-th/0701248.

[17] M. Kiermaier and Y. Okawa, Exact marginality in open string field theory: a general framework, JHEP 0801 (2008) 028. [hep-th/0701249 [HEP-TH]].

[18] E. Fuchs, M. Kroyter and R. Potting, Marginal deformations in string field theory, arXiv:0704.2222 [hep-th].

[19] B. H. Lee, C. Park and D. D. Tolla, Marginal Deformations as Lower Dimensional D-brane Solutions in Open String Field theory, arXiv:0710.1342 [hep-th].

[20] O. K. Kwon, Marginally Deformed Rolling Tachyon around the Tachyon Vacuum in Open String Field Theory, Nucl. Phys. B 804, 1 (2008) [arXiv:0801.0573 [hep-th]].

[21] Y. Okawa, Analytic solutions for marginal deformations in open superstring field theory, arXiv:0704.0936 [hep-th].

[22] Y. Okawa, Real analytic solutions for marginal deformations in open superstring field theory, arXiv:0704.3612 [hep-th].

[23] M. Kiermaier and Y. Okawa, General marginal deformations in open superstring field theory, arXiv:0708.3394 [hep-th].

[24] T. Erler, Marginal Solutions for the Superstring, JHEP 0707 (2007) 050 [arXiv:0704.0930 [hep-th]].

[25] E. Fuchs and M. Kroyter, Analytical Solutions of Open String Field Theory, arXiv:0807.4722 [hep-th].

[26] M. Schnabl, Algebraic solutions in Open String Field Theory - a lightning review, arXiv:1004.4858 [hep-th].

[27] L. Rastelli and B. Zwiebach, Tachyon potentials, star products and universality, JHEP 0109, 038 (2001) [arXiv:hep-th/0006240].

[28] M. Schnabl, "Wedge states in string field theory," JHEP 0301 (2003) 004 [arXiv:hep-th/0201095].

[29] L. Bonora, C. Maccaferri, R. J. Scherer Santos and D. D. Tolla, Ghost story. I. Wedge states in the oscillator formalism, JHEP 0709, 061 (2007) [arXiv:0706.1025 [hep-th]]. 
[30] L. Bonora, C. Maccaferri, R. J. Scherer Santos and D. D. Tolla, Ghost story. II. The midpoint ghost vertex, JHEP 0911, 075 (2009) [arXiv:0908.0055 [hep-th]].

[31] L. Bonora, C. Maccaferri and D. D. Tolla, Ghost story. III. Back to ghost number zero, JHEP 0911, 086 (2009) [arXiv:0908.0056 [hep-th]].

[32] I. Affleck and A. W. W. Ludwig, Universal noninteger 'ground state degeneracy' in critical quantum systems, Phys. Rev. Lett. 67 (1991) 161.

[33] J. A. Harvey, D. Kutasov and E. J. Martinec, On the relevance of tachyons, arXiv:hep-th/0003101.

[34] S. Elitzur, E. Rabinovici and G. Sarkissian, On least action D-branes, Nucl. Phys. B 541 (1999) 246 [arXiv:hep-th/9807161].

[35] N. Moeller, A. Sen and B. Zwiebach, D-branes as tachyon lumps in string field theory, JHEP 0008 (2000) 039 [arXiv:hep-th/0005036].

[36] P. Fendley, F. Lesage and H. Saleur, Solving 1-d plasmas and 2-d boundary problems using Jack polynomials and functional relations, J. Statist. Phys. 79 (1995) 799 [arXiv:hep-th/9409176].

[37] P. Fendley, H. Saleur and N. P. Warner, Exact Solution Of A Massless Scalar Field With A Relevant Boundary Interaction, Nucl. Phys. B 430 (1994) 577 [arXiv:hep-th/9406125].

[38] M. Kiermaier, Y. Okawa, P. Soler, "Solutions from boundary condition changing operators in open string field theory," JHEP 1103 (2011) 122. [arXiv:1009.6185 [hep-th]].

[39] I. Ellwood, Singular gauge transformations in string field theory, arXiv:0903.0390 [hep-th].

[40] L. Rastelli, A. Sen and B. Zwiebach, Boundary CFT construction of D-branes in vacuum string field theory, JHEP 0111 (2001) 045 [arXiv:hep-th/0105168].

[41] I. Ellwood and M. Schnabl, Proof of vanishing cohomology at the tachyon vacuum, JHEP 0702 (2007) 096 [arXiv:hep-th/0606142].

[42] M. R. Gaberdiel and B. Zwiebach, "Tensor constructions of open string theories I: Foundations," Nucl. Phys. B 505 (1997) 569 [arXiv:hep-th/9705038].

[43] I. Ellwood, The closed string tadpole in open string field theory, JHEP 0808 (2008) 063 [arXiv:0804.1131 [hep-th]].

[44] J. A. Harvey, S. Kachru, G. W. Moore and E. Silverstein, Tension is dimension, JHEP 0003, 001 (2000) [arXiv:hep-th/9909072].

[45] E. Witten, Some computations in background independent off-shell string theory, Phys. Rev. D 47, 3405 (1993) [arXiv:hep-th/9210065].

[46] D. Kutasov, M. Marino and G. W. Moore, Some exact results on tachyon condensation in string field theory, JHEP 0010, 045 (2000) [arXiv:hep-th/0009148].

[47] M. Kiermaier, Y. Okawa and B. Zwiebach, The boundary state from open string fields, arXiv:0810.1737 [hep-th].

[48] D. Gaiotto and L. Rastelli, "Experimental string field theory," JHEP 0308 (2003) 048 [arXiv:hep-th/0211012].

[49] M. Kroyter, On string fields and superstring field theories, arXiv:0905.1170 [hep-th]. 
[50] M. Kroyter, Comments on superstring field theory and its vacuum solution, arXiv:0905.3501 [hep-th].

[51] M. Kroyter, Superstring field theory in the democratic picture, arXiv:0911.2962 [hep-th].

[52] I. Y. Aref'eva, R. V. Gorbachev and P. B. Medvedev, Tachyon Solution in Cubic Neveu-Schwarz String Field Theory, Theor. Math. Phys. 158 (2009) 320 [arXiv:0804.2017 [hep-th]].

[53] I. Y. Aref'eva and R. V. Gorbachev, On Gauge Equivalence of Tachyon Solutions in Cubic Neveu-Schwarz String Field Theory, arXiv:1004.5064 [hep-th].

[54] T. Erler, Exotic Universal Solutions in Cubic Superstring Field Theory, arXiv:1009.1865 [hep-th].

[55] T. Erler, C. Maccaferri, "Comments on Lumps from RG flows,” JHEP 1111 (2011) 091 [arXiv:1105.6057 [hep-th]].

[56] L. Bonora, S. Giaccari, D. D. Tolla, "The energy of the analytic lump solution in SFT," JHEP 1108 (2011) 158. [arXiv:1105.5926 [hep-th]].

[57] L. Bonora, S. Giaccari, D. D. Tolla, "Lump solutions in SFT. Complements," [arXiv:1109.4336 [hep-th]].

[58] L. Bonora, S. Giaccari, D. D. Tolla, "Analytic solutions for Dp branes in SFT," [arXiv:1106.3914 [hep-th]]. 